\title{
Generalized continuous and left-continuous t-norms arising from algebraic semantics for fuzzy logics *
}

\author{
Carles Noguera \\ Dept. of Mathematics and Computer Science, University of Siena \\ Pian dei Mantellini 44, 53100 Siena, Italy \\ cnoguera@iiia.csic.es \\ Francesc Esteva, Lluís Godo \\ Artificial Intelligence Research Institute (IIIA - CSIC) \\ Campus de la Universitat Autònoma de Barcelona s/n, \\ 08193 Bellaterra, Catalonia, Spain \\ \{esteva,godo\}@iiia.csic.es
}

\begin{abstract}
This paper focuses on the issue of how generalizations of continuous and leftcontinuous t-norms over linearly ordered sets should be from a logical point of view. Taking into account recent results in the scope of algebraic semantics for fuzzy logics over chains with a monoidal residuated operation, we advocate linearly ordered BL-algebras and MTL-algebras as adequate generalizations of continuous and left-continuous t-norms respectively. In both cases, the underlying basic structure is that of linearly ordered residuated lattices. Although the residuation property is equivalent to left-continuity in t-norms, continuous t-norms have received much more attention due to their simpler structure. We review their complete description in terms of ordinal sums and discuss the problem of describing the structure of their generalization to BL-chains. In particular we show the good behavior of BL-algebras over a finite or complete chain, and discuss the partial knowledge of rational BL-chains. Then we move to the general non-continuous case corresponding to left-continuous t-norms and MTL-chains. The unsolved problem of describing the structure of left-continuous t-norms is presented together with a fistful of construction-decomposition techniques that apply to some distinguished families of t-norms and, finally, we discuss the situation in the general study of MTL-chains as a natural generalization of left-continuous t-norms.
\end{abstract}

Keywords: BL-chains, Continuous t-norms, Left-continuous t-norms, Mathematical Fuzzy Logic, Monoidal t-norm based Logic (MTL), MTL-chains, MV-algebras, Rational chains.

\section{Introduction: t-norms and their generalizations}

Triangular norms (t-norms, for short) are a special kind of aggregation functions that appeared in the framework of probabilistic metric spaces (see Schweizer and Sklar's works

*This is a completely revised and extended version of the conference paper [27]. 
$[83,84])$ following the ideas of Menger exposed in [71] to deal with triangular inequality. They are binary functions defined over the real unit interval $T:[0,1]^{2} \rightarrow[0,1]$ such that for every $a, b, c \in[0,1]$ :

- $T(a, T(b, c))=T(T(a, b), c)$ (associativity)

- $T(a, b)=T(b, a)$ (commutativity)

- If $b \leq c$, then $T(a, b) \leq T(a, c)$ (monotonicity)

- $T(a, 1)=a$ (neutral element)

Some other properties follow immediately from the definition.

- $T(a, 0)=0$, for every $a \in[0,1]$.

- $T(a, b) \leq \min \{a, b\}$, for every $a, b \in[0,1]$.

Since they are binary functions we may use an infix operational notation, such as $a * b$, rather than $T(a, b)$.

In the late 70s and beginning of the 80s several scholars, like Alsina, Trillas and Valverde [3] Höhle [44], Klement [60] or Dubois and Prade [19, 20], proposed t-norms as a suitable class of functions to model the intersection of fuzzy sets (Fuzzy Set Theory had been founded by Zadeh in 1965 [89]), together with their dual functions, t-conorms, to model unions, and the so-called negation functions for the complement (see [85, 22]). As regards to implication, in [86] it was proposed to model it by using either R-implications or S-implications. Namely, given a t-norm $*$ the corresponding $\mathrm{R}$-implication and S-implication are respectively defined as binary functions $\rightarrow_{R}^{*}$ and $\rightarrow_{S}^{*}$ such that, for every $a, b \in[0,1]$,

$$
a \rightarrow{ }_{R}^{*} b=\sup \{c \in[0,1] \mid a * c \leq b\}
$$

and

$$
a \rightarrow{ }_{S}^{*} b=n(a * n(b))
$$

where $n$ is an involutive negation, i.e. a non-increasing bijection $n:[0,1] \rightarrow[0,1]$ such that $n(n(x))=x$ for all $x \in[0,1]$. These two implications are generalizations of the classical material implication when restricted to $\{0,1\}$. One advantage of R-implications is that they reflect the order, namely they satisfy the following property: $a \rightarrow_{R}^{*} b=1$ iff $a \leq b$, which is in the other hand not generally satisfied by S-implications.

In fact, the notion of R-implication is very close to the notion of residuum which arises from the theory of residuated lattices. Namely, an algebraic structure $\mathcal{A}=\langle A, \&, \rightarrow, \wedge, \vee, \overline{0}, \overline{1}\rangle$ is said to be a (bounded commutative integral) residuated lattice if:

- $\langle A, \wedge, \vee, \overline{0}, \overline{1}\rangle$ is a bounded lattice,

- $\langle A, \&, \overline{1}\rangle$ is an ordered commutative monoid with neutral element $\overline{1}$,

- $\rightarrow$ is the residuum of $\&$, or equivalently $(\&, \rightarrow)$ is an adjoint pair, i.e. for every $a, b, c \in A$ it holds that $a \& c \leq b$ if and only if $c \leq a \rightarrow b$. 
It is easy to see that, in a residuated lattice, the residuum is uniquely determined by the following stipulation: $a \rightarrow b=\max \{c \in A \mid a \& c \leq b\}$. This shows exactly the difference between an R-implication and a residuum in the case of t-norms. Indeed, since t-norms are defined over $[0,1]$, which is a complete set, the R-implication (the supremum) for each tnorm always exists, while the residuum (the maximum) in general may not exist. In fact, the residuum of a t-norm exists if, and only if, the t-norm is left-continuous.

In the last years, a great interest for left-continuous t-norms and their residua has come from the logical field. Indeed, as logical deductive systems were developed to reason with predicates that can be modeled by fuzzy sets, t-norms started to be used as a semantical interpretation of logical conjunction as well (and analogously for the remaining logical connectives and functions proposed to model set-theoric operations in fuzzy sets). In this new framework, whose landmark is Hájek's celebrated monograph [41], typically the particular fuzzy logics are syntactically presented as Hilbert-style calculi which afterwards receive a truth-functional correct and complete semantics formed by $[0,1]$-valued algebras. These logics often lack the structural law of contraction, which axiomatically can be expressed as $\varphi \rightarrow \varphi \& \varphi$, and this justifies their inclusion in the broad family of so-called substructural logics. ${ }^{1}$

As it is usual in these logics, the lack of contraction implies a splitting of conjunction connective into two different ones, say $\&$ and $\wedge$, which retain separately some classical properties of conjunction. In the typical [0,1]-valued algebras for fuzzy logics \& is interpreted by a t-norm $*$, and $\wedge$ by the min function. Moreover, it is usual to have modus ponens as an inference rule in these systems: from $\varphi$ and $\varphi \rightarrow \psi$ infer $\psi$. Assume that $v$ is an evaluation of the formulae in $[0,1]$; by truth-functionality, the value of $\varphi \& \psi$ is $v(\varphi) * v(\psi)$ and the value of $\varphi \rightarrow \psi$ is $v(\varphi) \Rightarrow v(\psi)$, where $\Rightarrow$ is the functional interpretation of implication (which is in principle required to at least preserve the $\{0,1\}$ truth value combinations of classical material implication). To have modus ponens as a valid rule amounts to require that $v(\varphi)=1$ and $v(\varphi) \Rightarrow v(\psi)=1$ imply $v(\psi)=1$, i.e. that the truth-value 1 be preserved by modus ponens. But in fuzzy logic we usually want something else, that inference be truth preserving, in the sense that the truer the premises are, the truer the conclusion is. Assuming that $\Rightarrow$ captures the order $(x \Rightarrow y=1$ iff $x \leq y)$, this is achieved by requiring that the formula $\varphi \&(\varphi \rightarrow \psi) \rightarrow \psi$ be a tautology, which in a sense is declaring that the truth value of the set of premises is obtained by aggregating $v(\varphi)$ and $v(\varphi \rightarrow \psi)$ by means of the t-norm $*$. Indeed, under our assumptions, $v(\varphi \&(\varphi \rightarrow \psi) \rightarrow \psi)=1$ holds iff $v(\varphi) *(v(\varphi) \Rightarrow v(\psi)) \leq v(\psi)$. Although many definitions of $\Rightarrow$ would satisfy this condition, the traditional solution is to take $v(\varphi) \Rightarrow v(\psi)=\max \{c \in[0,1] \mid v(\varphi) * c \leq v(\psi)\}$, i.e. the maximum value that fulfills the required inequality, and so making the modus ponens rule as powerful as possible (in the sense that it will allow to derive maximally true conclusions). Obviously this condition requires $\Rightarrow$ to be the residuum of $*$-and not just its R-implication- and therefore $*$ must be left-continuous (we usually denote the residuum of $*$ by $\Rightarrow_{*}$ ). Thus, the typical $[0,1]$-valued semantics for fuzzy logics are algebras defined by a left-continuous t-norm; in symbols: algebras of the form $[0,1]_{*}=\left\langle[0,1], *, \Rightarrow_{*}\right.$, min, max, 0,1$\rangle$, which are a particular kind of residuated lattices. It explains, eventually, why left-continuous t-norms have become a central issue for Mathematical Fuzzy Logic (the subfield of Mathematical Logic devoted to the study of fuzzy logic systems, that, mainly arising from [41], has been rapidly growing in recent times).

In a first stage, fuzzy logics were based on semantics of continuous t-norms. It was the

\footnotetext{
${ }^{1}$ For an up-to-date monograph on substructural logics we refer the reader to [35].
} 
case of the main systems studied in [41] and in the pioneering works that led to Hájek's monograph: Łukasiewicz logic (complete w.r.t. Łukasiewicz t-norm [64, 10]), Gödel-Dummett logic (complete w.r.t. the t-norm given by the minimum operation $[38,21]$ ), Product (complete w.r.t. the t-norm given by the usual product of reals [43]) and BL logic (complete w.r.t. the semantics given by all continuous t-norms [41, 13]). In fact, the first example of a leftcontinuous non-continuous t-norm was found in the beginning of the nineties, namely the nilpotent minimum t-norm [81,31]. This finding, and the fact that left-continuity is the sufficient and necessary condition for a t-norm to have a residuum, motivated the introduction of MTL logic by Esteva and Godo [24] which was proved to be complete w.r.t. the semantics given by all left-continuous t-norms [57]. However, no matter how well justified the leftcontinuous t-norm based logics might be, there is a crucial difference between their study and that of logics of continuous t-norms: while the ordinal sum representation provides a nice and useful structural theorem for continuous t-norms [75, 63], we lack a general structural description of left-continuous t-norms.

Besides their origin in probabilistic metric spaces and their interest in Fuzzy Set Theory and in Mathematical Fuzzy logic, t-norms have proved to be useful aggregation operators in many other contexts, so their study has become an independent enterprise which has led to an intensive research (see for instance the monographs [61, 4]). Such development of tnorm theory has produced, in particular, several extensions of the initial notion of t-norm motivated by their applications. For instance, some researchers have extended the concept by replacing the domain $[0,1]$ by any finite totally ordered set, i.e. generalized t-norms over finite sets $[39,69,70]$. In particular, this trend has resulted in a valuable classification of the so-called smooth t-norms in [69] which is analogous to the representation of continuous t-norms. Another stream of research has consisted in further generalizing t-norms over direct product of lattices $[18,56]$, such as $[0,1]^{2}$ or in general $[0,1]^{n}$, or over arbitrary bounded partially ordered sets [90]. ${ }^{2}$ Finally, another enhancement of t-norm theory appears naturally when considering the algebraic semantics of fuzzy logic systems. A wide family of these logics, in addition to their possible completeness w.r.t. a semantics of left-continuous t-norms on $[0,1]$, are algebraizable in the sense that they enjoy a correct and complete algebraic semantics which is linked to the logic in a very strong way (see [6]). Algebraic semantics for fuzzy logics are typically a variety of algebras (a class that can be presented by means of equations) ${ }^{3}$ with two important features: (i) all its members are bounded commutative integral residuated lattices, which implies that they have an operation \& behaving essentially as a t-norm (associative, commutative, monotonic and with an neutral element which is the maximum of the lattice), and (ii) every algebra is representable as a subdirect product of linearly ordered algebras in the variety. Therefore, one can consider the monoidal operation $\&$ in these algebras as a natural generalization of t-norms over some special kind of lattices or, more restrictively, just t-norms over the linearly ordered algebras (as they are enough to describe all the members of the variety). Some recent works show that Mathematical Fuzzy Logic is starting to emancipate from the interval $[0,1]$ as the only intended semantics for the logics, and other semantics of linearly ordered algebras are being considered instead, such as those given by finite chains, chains over the rationals or chains over the hyperreals (see

\footnotetext{
${ }^{2}$ In this general setting the study of t-norms actually becomes exactly the study of bounded commutative partially ordered monoids.

${ }^{3}$ Equivalently, a variety is a class of algebras closed under homomorphic images, subalgebras and direct products. This or any other unexplained basic notion from Universal Algebra can be found for instance in the monograph [8].
} 
e.g. $[30,15,28,73])$. Notice, that this last direction differs from the previous mentioned generalizations in a crucial point: as long as it must provide a semantics for fuzzy logics it must preserve the residuation property.

The present paper is devoted to the generalizations of (left-continuous) t-norms in the latter sense, namely residuated commutative integral monoidal operations over chains. Moreover, the study of those structures has revealed and exploited deep connections with other well-known algebraic structures, such as lattice-ordered Abelian groups [76, 14]. Therefore, results and construction methods for those generalized t-norms can also be of interest not only for researchers on technical aspects of t-norms but also for algebraists working on related structures. Thus, this paper has these two intended audiences, and it can be considered both as a survey and a position paper. First, it has a survey nature because we will offer a summary of the state of the art on the study of linearly ordered algebras for fuzzy logics with a monoidal operation of the mentioned type. Second, our advocation of this particular kind of generalized t-norms as an interesting field from both the logical and algebraic points of view, with plenty of mathematically difficult and challenging open problems, makes it a position paper as well. Finally, the presentation will be also accompanied with some results, observations and examples that will complement the exposition of the problems.

The paper is organized as follows. After this introduction, Section 2 deals with continuous t-norms and their generalization, BL-chains. We summarize the complete knowledge of continuous t-norms and BL-chains over finite chains, present a general structural description of all BL-chains and discuss its limitations when trying to describe algebras over the rational unit interval. Section 3 deals with the much more difficult case of left-continuous t-norms and MTL-chains. We describe the partial available knowledge as regards to the attempts of understanding their structure and the methods to construct families of MTL-chains. Finally, in Section 4 we briefly discuss a potential alternative of linking the generalized notions of continuity and left-continuity to the order topology and show the problems of such an approach. We end up with some conclusions and remarks.

\section{Continuous t-norms and BL-chains}

\subsection{A refresher on the structure of continuous t-norms}

For the sake of the reader convenience, we start with recalling some basic facts on the structure of continuous t-norms. For full details, the reader is referred to the exhaustive monograph on t-norms [61]. First we collect in the following proposition some well-known basic properties of a t-norm and its residuum.

Proposition 2.1. Let $*$ be a t-norm. Then the following properties hold:

- $*$ has a residuum $\Rightarrow_{*}$ if, and only if, $*$ is left-continuous (Left-continuity)

- If $*$ has a residuum, then for every $a, b \in[0,1] a \leq b$ if, and only if, $a \Rightarrow_{*} b=1$ (Order and residuum)

- If $*$ has a residuum, then for every $a, b \in[0,1] \max \left\{a \Rightarrow_{*} b, b \Rightarrow_{*} a\right\}=1$ (Prelinearity)

- If $*$ has a residuum, then for every $a, b \in[0,1] \max \{a, b\}=\min \left\{\left(a \Rightarrow_{*} b\right) \Rightarrow_{*} b,\left(b \Rightarrow_{*}\right.\right.$ $\left.a) \Rightarrow_{*} a\right\}$ (Definability of maximum) 
- Assume $*$ is a left-continuous t-norm. Then, $*$ is continuous if, and only if, it is divisible (i.e. if $a<b$ there exists $c$ such that $a=b * c$ ), or equivalently, if and only if the t-norm and its residuum satisfy the divisibility equation: for every $a, b \in[0,1]$, $a *\left(a \Rightarrow_{*} b\right)=\min \{a, b\}$ (Divisibility)

Therefore, given a left-continuous t-norm $*$, the structure $[0,1]_{*}=\left\langle[0,1], *, \Rightarrow_{*}, \min , \max , 0,1\right\rangle$ is a residuated lattice satisfying the properties of prelinearity and definability of the maximum and, if $*$ is also continuous, the property of divisibility.

There are three main examples of continuous t-norms:

1. Eukasiewicz t-norm: $a *_{\mathrm{L}} b=\max \{0, a+b-1\}$

2. Product t-norm: $a *_{\Pi} b=a b$ (the usual product of real numbers)

3. Minimum (or Gödel) t-norm: $a *_{\mathrm{G}} b=\min \{a, b\}$

Their residua are respectively the following:

$$
\begin{aligned}
& a \Rightarrow_{\mathrm{七}} b= \begin{cases}1 & \text { if } a \leq b, \\
1-a+b & \text { otherwise. }\end{cases} \\
& a \Rightarrow_{\Pi} b= \begin{cases}1 & \text { if } a \leq b, \\
b / a & \text { otherwise. }\end{cases} \\
& a \Rightarrow_{\mathrm{G}} b= \begin{cases}1 & \text { if } a \leq b, \\
b & \text { otherwise. }\end{cases}
\end{aligned}
$$

The residuated lattices that they define are denoted respectively as $[0,1]_{\mathrm{L}},[0,1]_{\Pi}$ and $[0,1]_{\mathrm{G}}$.

They are the most prominent examples of continuous t-norms because it is possible to describe all continuous t-norms in terms of these three distinguished ones by using the notion of ordinal sum. This notion was born in the field of ordered semigroups (see $[16,17,33]$ ). In the particular case of t-norms, the definition is the following (see e.g. [49]):

Definition 2.2. Let $\left\{\left[a_{i}, b_{i}\right] \mid i \in I\right\}$ be a countable family of closed subintervals of $[0,1]$ such that their interiors are pairwise disjoint. For every $i \in I$, let $*_{i}$ be a $t$-norm defined on $\left[a_{i}, b_{i}\right]^{2}$. The ordinal sum of this family of t-norms is the operation defined as:

$$
x * y= \begin{cases}x *_{m} y & \text { if } \exists m \in I \text { such that } x, y \in\left[a_{m}, b_{m}\right], \\ \min \{x, y\} & \text { otherwise. }\end{cases}
$$

It is straigthforward to prove that the ordinal sum of a family of continuous t-norms is a continuous t-norm, and the ordinal sum of a family of left-continuous t-norms is a leftcontinuous t-norm. In the following we briefly sketch how (independently) Mostert and Shields ([75]) and Ling ([63]) obtained a description of continuous-torms in terms of ordinal sums of the three basic ones.

Proposition 2.3. Let $*$ be a continuous t-norm and $u \in[0,1]$ an idempotent element (i.e. $u * u=u)$. Then, for every $a, b \in[0,1]$ such that $a \leq u \leq b$, we have $a * b=a$. Therefore, the restrictions of $*$ to $[0, u]^{2}$ and to $[u, 1]^{2}$ are continuous t-norms and $*$ is their ordinal sum. 
Theorem 2.4 ([75, 63]). Let $*$ be a continuous t-norm. Then the set of its idempotent elements is a closed subset of $[0,1]$, and thus, its complement is the union of a countable family of pairwise disjoint open intervals. Moreover, if $\mathcal{I}(*)$ denotes the family of the closures of these intervals, then:

(i) For every interval $I \in \mathcal{I}(*)$, the restriction of $*$ to $I^{2}$ is isomorphic to $*_{\Pi}$ if it does not have any nilpotent element, or to $*_{\mathrm{L}}$ otherwise.

(ii) If $a, b \in[0,1]$ and there is no $I \in \mathcal{I}(*)$ such that $a, b \in I$, then $a * b=\min \{a, b\}$.

Thus, every continuous t-norm can be decomposed as an ordinal sum of the three basic ones.

\subsection{BL-chains as a generalization of continuous t-norms}

By Proposition 2.1, continuous t-norms are algebraically characterized by residuation and divisibility properties. Following this idea and with the goal of obtaining an algebraic semantics for BL logic, Hájek introduces in [41] BL-algebras as bounded commutative integral residuated lattices satisfying the following two equations:

- Prelinearity: $(x \rightarrow y) \vee(y \rightarrow x) \approx \overline{1}$.

- Divisibility: $x \&(x \rightarrow y) \approx x \wedge y$.

Thus, the algebraic structures defined by continuous t-norms are exactly the BL-algebras over $[0,1]$. There are some distinguished subclasses of BL-algebras:

Definition 2.5. Let $\mathcal{A}$ be a BL-algebra.

- $\mathcal{A}$ is an MV-algebra if it satisfies the involution equation: $x \approx(x \rightarrow \overline{0}) \rightarrow \overline{0}$.

- $\mathcal{A}$ is a П-algebra if it satisfies the cancellativity equation: $(y \rightarrow \overline{0}) \vee((y \rightarrow x \& y) \rightarrow x) \approx$ $\overline{1}$.

- $\mathcal{A}$ is a $\mathrm{G}$-algebra if it satisfies the contraction equation: $x \approx x \& x$.

Since they equationally given, these classes of algebras are in fact varieties. Observe that $[0,1]_{\mathrm{L}},[0,1]_{\Pi}$ and $[0,1]_{\mathrm{G}}$ are respectively an MV-algebra, a $\Pi$-algebra and G-algebra.

The property of prelinearity allows to prove the following proposition which shows that the study of BL-algebras (and any of their subvarieties such as MV-algebras, П-algebras or G-algebras) can be reduced to the study of the linearly ordered ones.

Proposition 2.6 ([41]).

- Every BL-algebra is a subdirect product of linearly ordered BL-algebras (which we will call BL-chains).

- Every variety of BL-algebras is generated by its linearly ordered members (its chains).

We can see the monoidal operation of BL-chains as a natural generalization of the notion of continuous t-norm over arbitrary totally ordered sets. In order to deal with this kind of structures it will be very useful to recall a couple of constructions that show a strong relation between Abelian $\ell$-groups and both MV-algebras and $\Pi$-algebras: 
1. Mundici's $\boldsymbol{\Gamma}$ functor: Chang introduced in [9] a certain transformation to show an equivalence between MV-chains and totally ordered Abelian groups. Mundici extended the construction in [76] to a functor that yields an equivalence of categories between the category of Abelian $\ell$-groups with strong unit and the category of MV-algebras. It is defined as follows. Given an Abelian $\ell$-group $\mathcal{G}=\langle G, \wedge, \vee,+,-, 0\rangle$ and a strong unit $u \in G$ (i.e. an element $u \geq 0$ such that for every $g \in G$ there exists a natural number $n \geq 1$ such that $g \leq n u)$, it is defined an MV-algebra $\boldsymbol{\Gamma}(\mathcal{G}, u)=\langle\{a \in G \mid 0 \leq a \leq$ $u\}, \&, \rightarrow, \wedge, \vee, 0, u\rangle$ with the operations:

$$
\begin{aligned}
a \& b & =0 \vee(a+b-u) \\
a \rightarrow b & =u \wedge(u-a+b) .
\end{aligned}
$$

Moreover, it is proved that for every MV-algebra $\mathcal{A}$ there is a unique (up to isomorphism) Abelian $\ell$-group with strong unit $\langle\mathcal{G}, u\rangle$ such that $\mathcal{A} \cong \Gamma(\mathcal{G}, u)$. For instance, if $\mathcal{R}$ denotes the additive group of reals, we have $[0,1]_{\mathrm{E}}=\boldsymbol{\Gamma}(\mathcal{R}, 1)$.

2. Cignoli-Torrens' $\mathbf{N}$ functor: Similarly to the previous case, in [14] it is introduced a functor that yields an equivalence of categories between the category of Abelian $\ell$-groups and a certain (full) subcategory of $\Pi$-algebras, ${ }^{4}$ which contains all linearly ordered $\Pi$ algebras. It is defined as follows. Given an Abelian $\ell$-group $\mathcal{G}=\langle G, \wedge, \vee,+,-, 0\rangle$ its negative cone is the set $G^{-}=\{a \in G \mid a \leq 0\}$. We add to a $G^{-}$a new element $\perp$ and define a $\Pi$-algebra $\mathbf{N}(\mathcal{G})=\left\langle G^{-} \cup\{\perp\}, \&, \rightarrow, \wedge, \vee, \perp, 0\right\rangle$ where:

$$
\begin{aligned}
& a \& b= \begin{cases}a+b & \text { if } a, b \in G^{-}, \\
\perp & \text { otherwise. }\end{cases} \\
& a \rightarrow b= \begin{cases}0 \wedge(b-a) & \text { if } a, b \in G^{-} \\
0 & \text { if } a=\perp, \\
\perp & \text { if } a \in G^{-} \text {and } b=\perp\end{cases}
\end{aligned}
$$

Then we have that for every $\Pi$-algebra $\mathcal{A}$ in the subcategory (hence for every $\Pi$-chain in particular), there is a unique (up to isomorphism) Abelian $\ell$-group $\mathcal{G}$ such that $\mathcal{A} \cong \mathbf{N}(\mathcal{G})$. For instance, $[0,1]_{\Pi} \cong \mathbf{N}(\mathcal{R})$.

To study the structure of BL-chains we also have at our disposal two decomposition theorems. The first one is based on the already seen decomposition of a continuous t-norm as ordinal sum of the three basic continuous t-norms. Namely, this decomposition has been generalized in $[42,13]$ by showing that every BL-chain is an ordinal sum of MV-chains, $\Pi$ chains and G-chains, or a subalgebra of such an ordinal sum obtained by removing some idempotent elements.

The second decomposition theorem for BL-chains states that every BL-chain is an ordinal sum of Wajsberg hoops and, although it is less known, it has some advantages so we will next summarize it. All results of this section about hoops and the decomposition theorem are taken from $[5,2,1]$.

\footnotetext{
${ }^{4}$ Actually, it is the subcategory of those $\Pi$-algebras $\mathcal{A}$ satisfying the following condition: for each $x \in A$, if $x>\overline{0}$ then $\neg x=0$.
} 
Definition 2.7. An algebraic structure $\mathcal{A}=\langle A, \&, \rightarrow, \overline{1}\rangle$ is a hoop if \& is a commutative monoidal operation with $\overline{1}$ as neutral element, and $\rightarrow$ is a binary operation satisfying for all $a, b, c \in A$ :
(i) $\quad a \rightarrow a=\overline{1}$,
(ii) $\quad a \&(a \rightarrow b)=b \&(b \rightarrow a)$,
(iii) $\quad a \& b \rightarrow c=a \rightarrow(b \rightarrow c)$

Moreover, an order relation on $\mathcal{A}$ can be defined as: $a \leq b$ iff $a \rightarrow b=\overline{1}$. $A$ hoop $\mathcal{A}$ is called prelinear. ${ }^{5}$ if it further satisfies:

(iv) $(a \rightarrow b) \rightarrow c \leq((b \rightarrow a) \rightarrow c) \rightarrow c$

$A$ hoop $\mathcal{A}$ is Wajsberg if $(a \rightarrow b) \rightarrow b=(b \rightarrow a) \rightarrow a$ for every $a, b \in A$. $\mathcal{A}$ is cancellative if $a \& b \leq c \& b$ implies $a \leq c$, for every $a, b, c \in A$. $\mathcal{A}$ is bounded if it has a minimum element, and unbounded otherwise. $\mathcal{A}$ is said to be weakly cancellative if it is either cancellative or it is bounded with a minimum element $\overline{0}$ and $a \& b \leq c \& b \neq \overline{0}$ implies $a \leq c$, for every $a, b, c \in A$.

Cancellative hoops are exactly unbounded Wajsberg hoops, and all Wajsberg hoops are prelinear. The order of a prelinear hoop defines a lattice structure where $a \wedge b=a \&(a \rightarrow b)$ and $a \vee b=((a \rightarrow b) \rightarrow b) \wedge((b \rightarrow a) \rightarrow a)$. Observe that all linearly ordered hoops are prelinear. ${ }^{6}$ Moreover, an easy computation shows that $\rightarrow$ is the residuum of $\&$. In fact, a prelinear hoop satisfies the same conditions than a BL-algebra except for the lower bound whose existence is not postulated in a hoop. For example, in the corresponding fragment of the language, if we remove the bottom element in $[0,1]_{\Pi}$ we obtain a cancellative hoop, and if we remove bottom element bound in $[0,1]_{\mathrm{G}}$ we obtain a prelinear hoop. Observe that any BL-chain is, actually, a bounded prelinear hoop; in particular (again in the $\overline{0}$-free fragment of the language) $[0,1]_{\mathrm{L}}$ is a bounded totally ordered Wajsberg hoop.

The notion of ordinal sum also extends to hoops but it needs some modification. It was first introduced in [29] for ordinal sums of two hoops and then generalized in [2] to sums of arbitrary families of hoops.

Definition 2.8. Let $\langle I, \leq\rangle$ be a totally ordered set. For all $i \in I$, let $\mathcal{A}_{i}$ be a totally ordered hoop such that for $i \neq j, A_{i} \cap A_{j}=\{\overline{1}\}$. Then $\bigoplus_{i \in I} \mathcal{A}_{i}$ (the ordinal sum of the family $\left.\left\{\mathcal{A}_{i} \mid i \in I\right\}\right)$ is the structure whose universe is $\bigcup_{i \in I} A_{i}$ and whose operations are:

$$
\begin{gathered}
a \& b= \begin{cases}a \&^{\mathcal{A}_{i} b} & \text { if } a, b \in A_{i}, \\
b & \text { if } a \in A_{i} \text { and } b \in A_{j} \backslash\{\overline{1}\} \text { with } i>j, \\
a & \text { if } a \in A_{i} \backslash\{\overline{1}\} \text { and } b \in A_{j} \text { with } i<j .\end{cases} \\
a \rightarrow b= \begin{cases}a \rightarrow^{\mathcal{A}_{i}} b & \text { if } a, b \in A_{i}, \\
b & \text { if } a \in A_{i} \text { and } b \in A_{j} \text { with } i>j, \\
\overline{1} & \text { if } a \in A_{i} \backslash\{\overline{1}\} \text { and } b \in A_{j} \text { with } i<j .\end{cases}
\end{gathered}
$$

For every $i \in I, \mathcal{A}_{i}$ is called a component of the ordinal sum.

\footnotetext{
${ }^{5}$ In the literature these structures were originally called basic hoops. However, since the relevant property in defining this class of hoops is prelinearity, we prefer to call them prelinear hoops.

${ }^{6} \mathrm{An}$ easy computation shows that this prelinearity condition, after the definition of the order, is equivalent to the prelinearity condition on the t-norm setting, i.e. to the condition $(a \rightarrow b) \vee(b \rightarrow a)=\overline{1}$ for all $a, b \in A$.
} 
Definition 2.9. A totally ordered hoop is indecomposable if it is not isomorphic to any ordinal sum of two non-trivial totally ordered hoops.

Observe that the difference between this notion of ordinal sum and the previous one is the identification of the top element of each component in a unique top element of the ordinal sum. It is also important to notice that in this case the components of the decomposition are subalgebras of the ordinal sum, which was not true in the original notion of ordinal sum.

Now we turn our attention to Wajsberg hoops since they play a basic role in the decomposition theorem. Basic examples of linearly ordered Wajsberg hoops are:

- $\mathbf{E}$, defined over $[0,1]$ by the Eukasiewicz t-norm and its residuum (bounded),

- $\mathbf{C}$, defined over $(0,1]$ by the product t-norm and its residuum (cancellative and unbounded), and

- 2, the two-element Boolean algebra defined over $\{0,1\}$.

It is clear that $\mathbf{E}, \mathbf{C}$ and $\mathbf{2}$ are indecomposable Wajsberg hoops. Actually, this is a property of any of the so-called Archimedean hoops, which are defined next using the following notion of Archimedean component (see e.g. [33]).

Definition 2.10. Let $\mathcal{A}$ be a totally ordered hoop and let $\sim$ be the following relation:

$$
a \sim b \text { if, and only if, for some } n \in \mathbb{N} \text {, either } a^{n} \leq b \leq a \text { or } b^{n} \leq a \leq b
$$

Then $\sim$ is an equivalence relation and the corresponding classes are called Archimedean components. The component containing the element $a \in A$ will be denoted by $[a]_{\sim}$.

Definition 2.11. A totally ordered hoop $\mathcal{A}$ is Archimedean if their Archimedean components are just $\{\overline{1}\}$ and $A \backslash\{\overline{1}\}$.

The following straightforward proposition, which is part of the folklore, gives the indecomposability of not only $\mathbf{E}, \mathbf{C}$ and $\mathbf{2}$ but of all totally ordered Archimedean hoops:

Proposition 2.12. Let $\mathcal{A}$ be a totally ordered hoop. If $\mathcal{A}$ is Archimedean, then it is indecomposable.

Next proposition contains some basic properties of Wajsberg hoops.

Proposition 2.13. For every Wajsberg hoop $\mathcal{A}$ the following conditions hold:

- (1) If $\mathcal{A}$ is bounded, then it is the $\overline{0}$-free reduct of an MV-algebra and it is weakly cancellative.

- (2) If $a \in A \backslash\{\overline{1}\}$ is an idempotent element, then it is the bottom element of the hoop.

- (3) The monoidal operation is continuous with respect to the order topology, i.e. if $\left\{a_{i} \mid i \in \mathbb{N}\right\}$ is a monotonic sequence in $\mathcal{A}$ that has limit $\alpha \in A$, then for every $b \in A$ :

(i) if the sequence is non-increasing (i.e. $\left.\alpha=\bigwedge a_{i}\right)$ then $\bigwedge\left(b \& a_{i}\right)$ exists and $b \& \alpha=$ $\bigwedge_{i}\left(b \& a_{i}\right)$,

(ii) if the sequence is non-decreasing (i.e. $\alpha=\bigvee a_{i}$ ) then $\bigvee\left(b \& a_{i}\right)$ exists and $b \& \alpha=$ $\bigvee_{i}\left(b \& a_{i}\right)$. 
Proof. (1) is obvious. To prove (2) take into account that if an idempotent element $a$ is not the minimum, there must exists an element $b<a$ and thus $(a \rightarrow b) \rightarrow b=\overline{1}$ and $(b \rightarrow a) \rightarrow a=a$ in contradiction with the equation defining Wajsberg hoops. As regards to (3), if $\alpha$ belongs to the sequence, the result is obvious. If the sequence is non-decreasing then the result is also true because \& is infinitely distributive with respect to $\vee$. Suppose that the sequence is non-increasing, $\alpha$ does not belong to the sequence and the conclusion is not true, i.e. $b \& \alpha<\bigwedge\left(b \& a_{i}\right)=d>0$ (obviously it is impossible to be greater). By divisibility there must exist an element $\beta$ such that both $b \& \beta=d$, and by weak cancellation this implies that $\alpha<\beta<a_{i}$ for all $a_{i}$ belonging to the sequence, which is impossible.

Notice that the proposition implies in particular that all Wajsberg hoops are weakly cancellative. We can state now the main result in [2] which is the decomposition theorem.

Theorem 2.14 ([2]). Every BL-chain is an ordinal sum of (indecomposable) linearly ordered Wajsberg hoops.

Let us compare now the decomposition based on the previous theorem with the previous one in the case of standard BL-chains, i.e. the BL-chains defined over $[0,1]$ by a continuous t-norm and its residuum.

1. For the three prominent continuous t-norms we have:

- $[0,1]_{\mathrm{E}}$ is an indecomposable Wajsberg hoop,

- $[0,1]_{\Pi}$ is isomorphic to the ordinal sum of $\mathbf{2}$ and $\mathbf{C}$, and

- $[0,1]_{\mathrm{G}}$ is isomorphic to the ordinal sum of as many $\mathbf{2}$ components as elements in $[0,1]$.

2. If $*$ is an arbitrary continuous t-norm, then its decomposition as ordinal sum of Wajsberg hoops contains (in the obvious order) as many components $\mathbf{E}$ as Łukasiewicz components appear in the usual decomposition, as many $\mathbf{C}$ components as Product components appear in the usual decomposition and as many $\mathbf{2}$ components as idempotent elements different from the top element the t-norm $*$ has.

Example 2.15. If $*$ is the ordinal sum (in the sense of t-norms) of a Eukasiewicz, a Product and a Gödel component in this order, then the decomposition as Wajsberg hoops is $[0,1]_{*} \cong$ $\mathbf{L} \oplus \mathbf{2} \oplus \mathbf{C} \oplus \bigoplus_{i \in 2^{\omega}} \mathbf{2}$.

Therefore, the indecomposable Wajsberg hoops possibly appearing in the decomposition of a BL-chain over the real unit interval are just isomorphic copies of $\mathbf{E}, \mathbf{C}$ and $\mathbf{2}$. Moreover, the components appearing in the decomposition are exactly the Archimedean components of the original standard BL-chain with the top element 1 . We will prove now that this situation generalizes to all BL-chains defined over a complete totally ordered set.

Proposition 2.16. Let $\mathcal{A}$ be a complete BL-chain and let $\mathcal{C}$ be a component of the decomposition of $\mathcal{A}$ as ordinal sum of indecomposable Wajsberg hoops. Then $\mathcal{C}$ is Archimedean.

Proof. First recall that, by (2) of Proposition 2.13, a Wajsberg hoop does not contain idempotent elements different from the bounds. 
Suppose that $\mathcal{C}$ has $\{\overline{1}\}$ (the trivial Archimedean component) and another Archimedean component $X \neq C \backslash\{\overline{1}\}$ which is not a singleton. If inf $X=\alpha \in C$ and this is not the infimum of $C$, then by (3) of Proposition 2.13 it is clear that $\alpha$ has to be an idempotent element and this is contradictory with our assumption. On the other hand if $\inf X=\inf C$ then by asssumption there must exist an element $b \in C \backslash\{\overline{1}\}$, such that $[\overline{1}]_{\sim}>[b]_{\sim}>X$ and thus $\inf [b]_{\sim} \in C$ and it is different from the infimum of $C$. Thus $\inf [b]_{\sim}$ must be idempotent which leads to a contradiction.

Corollary 2.17. Every complete BL-chain is decomposable as the ordinal sum of its Archimedean components.

We move now to the case of the rational unit interval. In that case the order of the chain is not complete and thus Proposition 2.16 does not apply. The following examples show that we can find indecomposable Wajsberg hoops over the rational unit interval which are not Archimedean.

Example 2.18. Let $\mathcal{H} \Pi$ be the hoop defined over $(0,1] \cap \mathbb{Q}$ by the usual product of rational numbers (i.e. the $\overline{0}$-free reduct of the standard $\Pi$-chain $[0,1]_{\Pi}$ restricted to the rationals without zero). Take a cut in the interior of the rational unit interval defined by an irrational $\alpha \in(0,1)$ and take the isomorphic copy of $\mathcal{H} \Pi$ over $\mathbb{Q} \cap(\alpha, 1]$ (denote by $\times$ its monoidal operation). Take an involutive negation $n: \mathbb{Q} \cap[0,1] \rightarrow \mathbb{Q} \cap[0,1]$ such that $n(\mathbb{Q} \cap(\alpha, 1])=\mathbb{Q} \cap[0, \alpha)$ and define the monoidal operation over $\mathbb{Q} \cap[0,1]$ as,

$$
x * y= \begin{cases}x \times y, & \text { if } x, y>\alpha \\ n(x \rightarrow \times n(y)), & \text { if } x>\alpha, \text { and } y<\alpha \\ n(y \rightarrow \times n(x)), & \text { if } x<\alpha, \text { and } y>\alpha \\ 0, & \text { otherwise }\end{cases}
$$

where $\rightarrow_{\times}$refers to the residuum of $\times$. It is an MV-chain. The elements of this algebra are divided in two groups: the so-called infinite elements (those belonging to $\mathbb{Q} \cap(\alpha, 1]$ ), which form a filter, and the so-called infinitesimal elements (the elements of $\mathbb{Q} \cap[0, \alpha)$ ) and $x * y=0$ for all infinitesimals $x, y$. Clearly, this Wajsberg hoop is indecomposable and has three Archimedean components: $\{1\}, \mathbb{Q} \cap(\alpha, 1)$ and $\mathbb{Q} \cap[0, \alpha) .^{7}$

Example 2.19. Take the lexicographic product of the additive ordered Abelian group of integers $\mathcal{Z}=\langle\mathbb{Z},+,-, 0, \leq\rangle$ and the multiplicative ordered Abelian group of positive rationals $\mathcal{Q}^{+}=\left\langle\mathbb{Q}^{+}, \cdot,()^{-1}, 1, \leq\right\rangle$, and denote it by $\mathcal{Z} \times$ lex $\mathcal{Q}^{+}$. Notice that its neutral element is $\langle 0,1\rangle$. Its negative cone, i.e. the restriction of the algebra over the elements which are smaller than the neutral element, ${ }^{8}$ is a cancellative Wajsberg hoop with three Archimedean components $\{\langle 0,1\rangle\},\{\langle 0, r\rangle \mid r \in \mathbb{Q} \cap(0,1]\}$ and $\left\{\langle-k, r\rangle \mid k \in \mathbb{N} \backslash\{0\}, r \in \mathbb{Q}^{+}\right\}$. Take finally a cut in $\mathbb{Q} \cap[0,1]$ defined by an irrational number $\alpha \in(0,1)$ and take the isomorphic transformation of the three Archimedean components into the three intervals $\{1\}, \mathbb{Q} \cap(\alpha, 1)$ and $\mathbb{Q} \cap(0, \alpha)$, respectively. The resulting structure is an indecomposable and cancellative Wajsberg hoop over the rational unit interval that has three Archimedean components.

\footnotetext{
${ }^{7}$ This example can be isomorphically presented by considering $\Gamma\left(\mathcal{Z} \times{ }_{\text {lex }} \mathcal{Q},\langle 1,0\rangle\right)$, where $\mathcal{Z}$ and $\mathcal{Q}$ denote respectively the additive groups of integers and rationals, and $\mathcal{Z} \times$ lex $\mathcal{Q}$ denotes their lexicographic product.

${ }^{8}$ Observe that by doing this we are performing the same operation as in the previously introduced functor $\mathbf{N}$ but without adding a bottom element.
} 
We focus now on the study of indecomposable Wajsberg hoops over the rational unit interval and specially on the characterization of those of them that are restrictions of indecomposable Wajsberg hoops over the reals.

Proposition 2.20. Let $\mathcal{A}$ be an Archimedean Wajsberg hoop over a countable chain. Then:

1. If $\mathcal{A}$ is bounded (i.e. the reduct of an MV-chain), then it is isomorphic to a subalgebra of $\mathbf{E}$.

2. If $\mathcal{A}$ is unbounded (i.e. a cancellative totally ordered hoop), then it is isomorphic to a subalgebra of $\mathbf{C}$.

Proof. Suppose that $\mathcal{A}$ is an MV-algebra. By Mundici's functor $\mathcal{A}$ is isomorphic to the MVchain $\boldsymbol{\Gamma}(\mathcal{G}, u)$ for some totally ordered Abelian group $\mathcal{G}$ and some strong unit $u \in G$. Actually (see [76]) they can be described in the following way. Recall that in an MV-algebra it is usual to define a negation operation as $\neg a=a \rightarrow \overline{0}$ and a dual operation of \& as $a \oplus b=\neg(\neg a \& \neg b)$. Then $\mathcal{G}$ is the linearly ordered Abelian group defined by:

- Universe: $G=\{\langle n, a\rangle \mid n \in \mathbb{Z}, a \in A \backslash\{\overline{0}\}\}$

- Order: the lexicographic order over $G$

- Operations: the sum is defined as,

$$
\langle n, a\rangle+\langle m, b\rangle= \begin{cases}\langle n+m, a \oplus b\rangle, & \text { if } a \oplus b<\overline{1} \\ \langle n+m+1, a \& b\rangle, & \text { otherwise }\end{cases}
$$

and the inverse is defined as,

$$
-\langle n, a\rangle= \begin{cases}\langle-n-1, \neg a\rangle, & \text { if } \overline{0}<a<\overline{1} \\ \langle-n, 0\rangle, & \text { if } a=\overline{0} .\end{cases}
$$

An easy computation shows that if $\mathcal{A}$ has only two Archimedean components, then the group $\mathcal{G}$ is Archimedean and thus by Hölder's Theorem (see e.g. [37]) it is isomorphic to a subgroup of the additive group $\mathcal{R}$ of the real numbers with the usual order and sum operation. Then $\mathcal{A}$ is isomorphic to a subalgebra of $\boldsymbol{\Gamma}(\mathcal{R}, 1)$ which coincides with the standard MV-chain over the real unit interval.

Suppose now that $\mathcal{A}$ is a cancellative hoop. Adapting slightly the functor $\mathbf{N}$ to cancellative hoops, we have that $\mathcal{A}$ is isomorphic to the negative cone of a linearly ordered Abelian group $\mathcal{G}$. Since $\mathcal{A}$ has only two Archimedean components, $\mathcal{G}$ must be an Archimedean group and thus, again by Hölder's Theorem, it is isomorphic to a subgroup of $\mathcal{R}$. Then the result just follows from the fact that the negative cone of $\mathcal{R}$ is isomorphic to $\mathbf{C}$.

Corollary 2.21. Let $\mathcal{A}$ be a Wajsberg hoop over a countable chain. $\mathcal{A}$ is Archimedean if, and only if, it is embeddable into $\mathbf{\mathbf { L }}$ or $\mathbf{C}$.

Nevertheless, while in the case of hoops over the reals there are, up to isomorphism, only three Archimedean Wajsberg hoops, in the rational case the situation is much more complex. Next we give examples of pairs of isomorphic Archimedean linearly ordered Abelian groups over the reals whose restrictions to the rationals are not isomorphic, even if they are still Archimedean. 
Example 2.22. Consider again the additive ordered group of reals $\mathcal{R}=\langle\mathbb{R},+,-, 0, \leq\rangle$ and the multiplicative group of positive reals $\mathcal{R}^{+}=\left\langle\mathbb{R}^{+}, \cdot,()^{-1}, 1, \leq\right\rangle$ with the natural total order as well. They are Archimedean and, as it is well known, isomorphic. Their restriction to the rationals are also Archimedean linearly ordered Abelian groups, $\langle\mathbb{Q},+,-, 0, \leq\rangle$ and $\left\langle\mathbb{Q}^{+}, \cdot,()^{-1}, 1, \leq\right\rangle$, but they are not isomorphic. The usual isomorphisms between the groups over the reals (the exponential and the logarithmic transformations) are not well defined over the rationals. Moreover, the fact that the additive group is divisible while the multiplicative is not ${ }^{9}$ implies that there cannot be such an isomorphism. By applying the functors $\boldsymbol{\Gamma}$ and $\mathbf{N}$ to these groups we obtain respectively an example in the case of bounded Wajsberg hoops and in the case of cancellative hoops of a pair of isomorphic Archimedean hoops over the reals that are not isomorphic anymore when restricted to the rationals.

The example suggests that, unlike what happens in the real case, there could be a huge number of non-isomorphic Archimedean Wajsberg hoops over the rationals. Therefore, we can consider the following interesting and difficult question:

Open problem: classify the isomorphism classes of Archimedean Wajsberg hoops over the rationals, or more generally, classify the isomorphism classes of indecomposable Wajsberg hoops over the rationals.

Of course, thanks to the representation in ordinal sums, a solution to this problem would bring a complete description of all possible generalizations of the notion of continuous tnorm over the rationals. Actually, due to Hölder's Theorem, the first part of the problem is equivalent to that of classifying dense subgroups of the additive ordered group of the reals $\mathcal{R}$. It is well-known (see e.g. [40, Lemma 4.21]) that subgroups of $\mathcal{R}$ are either dense or discrete (and then isomorphic to the subgroup of integers). For those dense groups which are subgroups of the additive ordered group of the rationals $\mathcal{Q}$ there is a classification due to Bauer (see e.g. [32, Section 42]). Therefore, it only remains the case of dense subgroups of $\mathcal{R}$ which are not subgroups of $\mathcal{Q}$. Here an algebraic classification of their isomorphism classes is not available, but we can still know something about the finitely generated ones. Indeed, since they are torsion-free, their reducts in the language of groups (i.e. disregarding the order) are always isomorphic to $\mathcal{Z}^{r}=\left\langle\mathbb{Z}^{r},+,-, \mathbf{0}\right\rangle$ for some $r \geq 1$ (see e.g. [32, Theorem 10.4]), and hence the problem corresponds to that of describing all possible Archimedean total orders on $\mathcal{Z}^{r}$. Now all such orders, whether Archimedean or not, are described in [62, Section 6.3]. On the other hand, the dense 1-generated subalgebras of the standard MV-algebra $[0,1]_{\mathrm{L}}$ are described in [34], showing that the subalgebra $\langle a\rangle$ generated by an irrational number $a$ is dense and $\langle a\rangle \cong\langle b\rangle$ for an irrational $b \neq a$ iff $a=1-b$. Thus there are uncountable many different 1-generated indecomposable Archimedean (bounded) Wajsberg hoops over the rationals.

As for the general non-Archimedean case, we can use Hahn's Theorem (see e.g. [37]) to conclude that indecomposable Wajsberg hoops over the rationals are still closely related to $\mathcal{R}$ by means of an embedding into a lexicographic product:

Theorem 2.23. Let $\mathcal{A}$ be a Wajsberg hoop over the rationals. Then:

1. If $\mathcal{A}$ is bounded (i.e. an $\mathrm{MV}$-chain), then it is embeddable into $\boldsymbol{\Gamma}\left(\prod_{i \in I}^{\text {lex }} \mathcal{R}, u\right)$, for some index set $I$.

\footnotetext{
${ }^{9}$ Recall that a group $\mathcal{G}=\left\langle G, \oplus,()^{-1}, e\right\rangle$ is said to be divisible if for every $x \in G$ and every natural number $n$ there is $y \in G$ such that $y \oplus \ldots{ }^{n} \oplus y=x$. This should not be confused with the previously introduced notion of divisible t-norm.
} 
2. If $\mathcal{A}$ is unbounded (i.e. a cancellative totally ordered hoop), then it is embeddable into $\mathbf{N}\left(\prod_{i \in I}^{l e x} \mathcal{R}\right)$, for some index set $I$.

where $\mathcal{R}=\langle\mathbb{R},+,-, 0, \leq\rangle$ is the additive ordered group of reals, $\prod_{i \in I}^{\text {lex }} \mathcal{R}$ denotes the lexicographic product of $I$ copies of this group, and $u=\langle 1,0, \ldots, 0\rangle$ (with I components) is a strong unit of $\prod_{i \in I}^{l e x} \mathcal{R}$.

Furthermore, if we further assume that $\mathcal{A}$ is finitely generated, then I can be taken with the same cardinal as the set of Archimedean components of $\mathcal{A}$.

Finally, we focus briefly on finite chains. As in the real case, the structure of such chains is completely known for BL-chains. They can be described just by using the first notion of ordinal sum (for BL-chains). Indeed, since there are no finite product chains (except for the trivial one and the two-element Boolean algebra, that obviously are MV and G-chains as well), all finite BL-chains are ordinal sums of finite MV and G-chains. Taking into account that finite MV-chains are isomorphic to the Eukasiewicz $n$-valued chains $\mathbf{L}_{n}$, and the obvious structure of finite Gödel chains $\mathcal{G}_{n}$, we obtain the following result (equivalent to the representation theorem for finite smooth t-norms given in [69]):

Proposition 2.24. Each finite BL-chain is isomorphic to an ordinal sum of a finite subfamily of $\left\{\mathbf{\mathbf { L }}_{n} \mid n \geq 1\right\} \cup\left\{\mathcal{G}_{n} \mid n \geq 1\right\}$.

Since for every $n \geq 2, \mathbf{E}_{n}$ (resp. $\mathcal{G}_{n}$ ) is a subalgebra of the MV-chain (resp. the G-chain) over the rational unit interval defined by the restriction of the Łukasiewicz t-norm (resp. the minimum t-norm), and hence it is also a subalgebra of $[0,1]_{\mathrm{E}}$ (resp. $[0,1]_{\mathrm{G}}$ ), every non-trivial finite BL-chain is embeddable both into a rational BL-chain and into a real BL-chain.

\section{Left-continuous t-norms and MTL-chains}

\subsection{The problem of classifying left-continuous t-norms}

We have argued in the introduction that residuation is an essential algebraic property that must be required to have good semantics for fuzzy logical systems based on modus ponens rule. We have also seen that the necessary and sufficient condition for a t-norm to have a residuum is not continuity but left-continuity. Therefore, it is definitely interesting from a logical point of view to focus on the study of left-continuous t-norms and their generalizations to arbitrary classes of linearly ordered sets.

Unfortunately, the knowledge on left-continuous t-norms is drastically limited in comparison to the nice description in the continuous case that we have seen. So far no structural theorem that would allow to classify them has been found and the only available pieces of knowledge are several families of left-continuous t-norms, some constructive techniques to obtain those families, some decomposition theorems (that are not strong enough to yield a classification) and some studies of particular features of these t-norms such as the description of their sets of continuity, sets of determination or their behavior under convex combinations. In fact, for a long period, the difficulty of the problem of finding a general description, in the style of Mostert and Shields theorem for continuous t-norms, has been perceived as nearly overwhelming; let us just quote Jenei's words from [54]: "the establishing of any structural theorem seems to be hopeless". Nevertheless, some very recent works suggest that there might be some room for hope. On the one hand, more general new construction and decomposition methods, encompassing the previously known ones, have appeared: the triple rotation 
method [65, 66, 67] and some generalizations of the rotation (-annihilation) methods [88]. On the other hand, in [87] a very general class t-norms, the so-called regular t-norms, has been proposed and deeply studied and, although is does not exhaust the class of all left-continous t-norms, is still a very powerful notion as we will discuss later. We will next review the state of the art on left-continuous t-norms by giving the basic notions and results, and referring the reader to the corresponding sources for a detailed exposition of each topic.

The first natural option one may consider when attempting to understand left-continuous t-norms is to extend the ordinal sum representation. The operation, of course, is also defined for such t-norms; but if we follow the proof of the representation theorem of continuous t-norms we soon realize that it cannot be generalized to the left-continuous case. Indeed, Proposition 2.3 already fails. Consider, for instance, Fodor's Nilpotent Minimum t-norm:

$$
a *_{\mathrm{NM}} b= \begin{cases}\min \{a, b\} & \text { if } a>1-b, \\ 0 & \text { otherwise. }\end{cases}
$$

Its residuum is:

$$
a \Rightarrow_{\mathrm{NM}} b= \begin{cases}1 & \text { if } a \leq b, \\ \max \{1-a, b\} & \text { otherwise. }\end{cases}
$$

Take, for example, 0.7. It is an idempotent element for $*_{\mathrm{NM}}$, but we have $x *_{\mathrm{NM}} 0.1=0$ for each $0.7 \leq x \leq 0.9$. Therefore, idempotent elements do not give in general a cut point to split a left-continuous t-norm as the ordinal sum of the piece below and the piece above. Nevertheless, one could still use Zorn's Lemma, and non-constructively prove that every leftcontinuous has a maximal decomposition as ordinal sum. The problem with this strategy is that there seems to be no way to classify the indecomposable pieces (in particular, all t-norms with an involutive negation are indecomposable and they form already a hardly describable class), so the decomposition becomes rather useless.

Having disregarded this dead-end street, scholars have concentrated on providing some techniques to obtain at least some families of left-continuous (and in general non continuous) t-norms. The main ones are the following:

1. Annihilation. Inspired by the definition of Nilpotent Minimum t-norm, Jenei introduced in [46] the annihilation technique. Given any continuous t-norm $*$ and an involution $n$ on $[0,1]$, he defined the $n$-annihilation of $*$ as the operation:

$$
a *_{n} b= \begin{cases}a * b & \text { if } a>n(b) \\ 0 & \text { otherwise. }\end{cases}
$$

This operation is not a t-norm in general. For instance, if $*=*_{\Pi}$ and $n(x)=1-x$, then $*_{n}$ is not a t-norm because it is not associative. Observe, on the other hand, that continuity in general will be lost, but left-continuity is preserved. In fact, in [46] it is proved that $*_{n}$ is a (left-continuous) t-norm iff it is isomorphic to $*_{\mathrm{NM}}$, to $*_{\mathrm{L}}$ or to the t-norm

$$
a \circ b= \begin{cases}0 & \text { if } a \leq 1-b \\ \frac{1}{3}+a+b-1 & \text { if } a, b \in\left[\frac{1}{3}, \frac{2}{3}\right] \text { and } a>1-b \\ \min \{a, b\} & \text { otherwise }\end{cases}
$$


In other words, this method allows to obtain an involutive left-continuous t-norm only if the initial one is an ordinal sum of $*_{\mathrm{G}}, *_{\mathrm{L}}$ and $*_{\mathrm{G}}$ in this order (the extremal cases are $*_{\mathrm{L}}$ which coincides with its own annihilation, and $*_{\mathrm{G}}$ whose annihilation is $*_{\mathrm{NM}}$ ).

Cignoli et al. [12] extended the method by allowing $n$ to be any weak negation function, non necessarily involutive. They describe the family of left-continuous t-norms that can be obtained in this way. In particular it contains the so-called Weak Nilpotent Minimum t-norms (introduced in [24]) which are the annihilations of $*_{\mathrm{G}}$ by arbitrary weak negation functions.

2. Rotation. A different method to obtain involutive left-continuous t-norms was introduced by Jenei in [47] in the following way. Let $*$ be a left-continuous t-norm such that either (i) it has no zero divisors, or (ii) there exists $c \in[0,1]$ such that for every zero divisor $a$ of $*, a \Rightarrow_{*} 0=c$. Let $n$ be an arbitrary involutive negation with fixpoint $\beta$, and $\sigma$ an arbitrary order-preserving bijection from $[\beta, 1]$ to $[0,1]$. With these ingredients, the rotation of $*$ is the binary operation:

$$
a \star b= \begin{cases}\sigma^{-1}(\sigma(a) * \sigma(b)), & \text { if } a, b>\beta \\ n\left(\sigma^{-1}\left(\sigma(a) \Rightarrow_{*} \sigma(n(b))\right)\right), & \text { if } a>\beta, b \leq \beta \text { and } n(a)<b \\ n\left(\sigma^{-1}\left(\sigma(b) \Rightarrow_{*} \sigma(n(a))\right)\right), & \text { if } a \leq \beta, b>\beta \text { and } n(a)<b \\ 0, & \text { otherwise }\end{cases}
$$

Then $\star$ is a left-continuous t-norm and $n$ is its negation function. For instance, $*_{\mathrm{NM}}$ can be obtained as the rotation of $*_{\mathrm{G}}$ w.r.t. the involution $n(x)=1-x$.

3. Rotation-annihilation. A sort of combination of the two previous methods was introduced by Jenei as well [48]. Let $*_{1}$ be a left-continuous t-norm. Let $*_{2}$ be an arbitrary involutive left-continuous t-norm and let $n$ be an arbitrary involutive negation with fixpoint $\beta, \alpha$ an arbitrary number in $[\beta, 1), \sigma$ an order-preserving bijection from $[\alpha, 1]$ to $[0,1]$, and $\rho$ an order-preserving bijection from $[n(\alpha), \alpha]$ to $[0,1]($ if $\alpha \neq \beta)$. Moreover we assume that (i) if $\alpha=\beta, *_{1}$ has either no zero divisors or there exists $c \in[0,1]$ such that for every zero divisor $a$ of $*_{1}, a \Rightarrow_{*_{1}} 0=c$, or (ii) if $\alpha \neq \beta, \rho \circ n \circ \rho^{-1}$ coincides with the negation of $*_{2}$ and $*_{1}$ has no zero divisors. With these ingredients, the rotation-annihilation of $*_{1}$ and $*_{2}$ is the binary operation:

$$
a \star b= \begin{cases}\sigma^{-1}\left(\sigma(a) *_{1} \sigma(b)\right), & \text { if } a, b \geq \alpha \\ n\left(\sigma^{-1}\left(\sigma(a) \Rightarrow_{*_{1}} \sigma(n(b))\right)\right), & \text { if } a \geq \alpha, b \leq n(\alpha) \text { and } n(a)<b \\ n\left(\sigma^{-1}\left(\sigma(b) \Rightarrow_{*_{1}} \sigma(n(a))\right)\right), & \text { if } a \leq n(\alpha), b \geq \alpha \text { and } n(a)<b \\ \rho^{-1}\left(\rho(a) *_{2} \rho(b)\right), & \text { if } a, b \in(n(\alpha), \alpha) \text { and } n(a)<b \\ a, & \text { if } a \in(n(\alpha), \alpha), b \geq \alpha \\ b, & \text { if } b \in(n(\alpha), \alpha), a \geq \alpha \\ 0, & \text { otherwise }\end{cases}
$$

Then $\star$ is a left-continuous t-norm and $n$ is its negation function. Observe that when $\alpha=\beta$ we have the rotation of $*_{1}$.

4. Triple rotation. In very recent works Maes and De Baets have introduced and studied a new construction method to obtain involutive left-continuous t-norms (see $[65,66]$ ). Let $*$ be an arbitrary left-continuous t-norm. The companion of $*$ is an auxiliar operation 
defined as $a *^{\prime} b=\sup \left\{c \in[0,1] \mid a \Rightarrow_{*} c \leq b\right\}$. Let $n$ be an arbitrary involutive negation with fixpoint $\beta$, and $\sigma$ an arbitrary order-preserving bijection from $[\beta, 1]$ to $[0,1]$. Furthermore, let $f:[0,1] \rightarrow[0,1]$ be the decreasing function defined by $f(x)=1$ whenever $x \in[0, \beta]$ and $f(x)=\sigma^{-1}(n(\sigma(x)))$ whenever $x \in(\beta, 1]$. If $*$ has zero divisors, assume that $*^{\prime}$ is commutative. With these ingredients, the triple rotation of $*$ is the binary operation:

$$
a \star b= \begin{cases}\sigma^{-1}(\sigma(a) * \sigma(b)), & \text { if } a, b>\beta \text { and } f(a)<b \\ n\left(\sigma^{-1}\left(\left(\sigma(a) \Rightarrow_{*} 0\right) *^{\prime}\left(\sigma(b) \Rightarrow_{*} 0\right)\right)\right), & \text { if } a, b>\beta \text { and } f(a) \geq b \\ n\left(\sigma^{-1}\left(\sigma(a) \Rightarrow_{*} \sigma(n(b))\right)\right), & \text { if } a>\beta, b \leq \beta \text { and } n(a)<b \\ n\left(\sigma^{-1}\left(\sigma(b) \Rightarrow_{*} \sigma(n(a))\right)\right), & \text { if } a \leq \beta, b>\beta \text { and } n(a)<b \\ 0, & \text { otherwise }\end{cases}
$$

Then $\star$ is a left-continuous t-norm and $n$ is its negation function. Observe that if $*$ has no zero divisors, then the case in the second line does never occur and the construction coincides with rotation: for instance $*_{\mathrm{NM}}$ is the triple rotation of $*_{\mathrm{G}}$ w.r.t. the involution $n(x)=1-x$. When applied to t-norms with zero divisors the method can yield already known involutive t-norms, such as $*_{\mathrm{E}}$ which is the triple rotation of itself w.r.t. the involution $n(x)=1-x$, or t-norms that cannot be obtained by means of the previous rotation and rotation-annihilation constructions such as the rotation of $*_{\mathrm{NM}}$ w.r.t. $n(x)=1-x$ :

$$
a \star b= \begin{cases}\min \{a, b\}, & \text { if } a, b>\frac{1}{2} \text { and } \frac{3}{2}-a<b \\ \frac{1}{2}, & \text { if } a, b>\frac{1}{2} \text { and } \frac{3}{2}-a \geq b \\ \min \left\{a-\frac{1}{2}, b\right\}, & \text { if } a>\frac{1}{2}, b \leq \frac{1}{2} \text { and } 1-a<b \\ \min \left\{b-\frac{1}{2}, a\right\}, & \text { if } a \leq \frac{1}{2}, b>\frac{1}{2} \text { and } 1-a<b \\ 0, & \text { otherwise }\end{cases}
$$

Conversely, these methods can also be seen as a way to decompose left-continuous into simpler components. Indeed, on the one hand, in [50] decomposition in terms of rotation and rotation-annihilation is considered; in particular the author shows that every involutive left-continuous t-norm $*$ is maximally decomposable as rotation-annihilation of some t-norms $*_{1}$ and $*_{2}$ (or just $*_{1}$ when rotation is enough), i.e. when taking the minimum $\alpha$ providing such a decomposition. As it happens with the ordinal sum decomposition, the limitation of this result is the lacking of any characterization of indecomposable elements (i.e. those t-norms $*$ admitting only the trivial decomposition, $*=*_{2}$ ). On the other hand, in [67] the authors use the triple rotation as an alternative decomposition method as well, and put it into a more general setting that allows to encompass this method together with Jenei's rotation and rotation-annihilation (see [67, Section 5.1]). Again, for the same reason, such a setting is still not enough to describe all involutive left-continuous t-norms.

Another remarkable recent approach to the problem is that of Vetterlein in [87]. The advantage of this approach is its wider scope, i.e. it does not deal only with involutive tnorms, but with the general problem of classifying all left-continuous t-norms. He defines a new technical notion, that of regular t-norm that allows to gain a valuable insight into the problem. Roughly speaking, a t-norm $*$ is regular if there is a natural number $n$ such that for every $a \in[0,1]$ the $a$-translation $\lambda_{a}(x)=x * a$ has at most $n$ discontinuity points, and $*$ satisfies some other additional simplicity requirements. The class of regular t-norms contains 
the basic continuous ones $\left(*_{\mathrm{L}}, *_{\Pi}\right.$ and $\left.*_{\mathrm{G}}\right)$ and it is closed under all the construction methods reviewed here (ordinal sum, annihilation, rotation, rotation-annihilation and triple rotation). Therefore, pretty much all known left-continuous t-norms are actually regular. ${ }^{10}$ The interest of this notion lies in the fact that they are enough to provide a complete semantics for MTL logic (technically: the class of standard algebras based on regular t-norms generates the variety of MTL-algebras) even if they are still simple enough to be described and characterized at a large extent.

Finally, other noteworthy developments are the following: study of $n$-contraction properties in left-continuous t-norms (algebraically defined by $x * \stackrel{n}{.} * * x=x * \stackrel{n-1}{*} * x$ ) and their corresponding logics $[11,45]$, the set of continuity points of a left-continuous t-norms $[59,55]$, subsets of $[0,1]^{2}$ that determine the structure of the whole left-continuous t-norm $[53,55]$, convex combinations of left-continuous t-norms [54], weak nilpotent minimum t-norms and their corresponding logics [80], cancellativity properties [68], extension of rotation and rotationannihilation methods to provide slightly bigger families of left-continuous t-norms [88], and a latter-day contribution in [82] where associativity of t-norms is characterized in terms of Reidemeister closure condition of 3 -webs and it yields yet a new method to construct leftcontinuous t-norms.

\subsection{MTL-chains as a generalization of left-continuous t-norms}

As we have seen, the algebraic structures that arise from the property of residuation are residuated lattices. In generalizing the notion of continuous t-norm one adds two properties to bounded commutative integral residuated lattices: prelinearity and divisibility. In fact, divisibility was only added to obtain continuity in the real case. Therefore, if we only want to obtain residuated structures over chains we do not need divisibility, just prelinearity. This motivates the definition of MTL-algebras as they were introduced in [24]:

Definition 3.1. An algebraic structure $\mathcal{A}=\langle A, \&, \rightarrow, \wedge, \vee, \overline{0}, \overline{1}\rangle$ is said to be an MTL-algebra if:

- $\langle A, \wedge, \vee, \overline{0}, \overline{1}\rangle$ is a bounded lattice,

- $\langle A, \&, \overline{1}\rangle$ is an ordered commutative monoid with neutral element $\overline{1}$,

- $\rightarrow$ is the residuum of \& (or \& and $\rightarrow$ form and adjoint pair), i.e. for every $a, b, c \in A$ : $a \& c \leq b$ if and only if $c \leq a \rightarrow b$.

- For every $a, b \in A,(a \rightarrow b) \vee(b \rightarrow a)=\overline{1}$.

A negation operation $\neg$ is defined by putting, for every $a \in A, \neg a=a \rightarrow \overline{0}$. If $\neg$ is involutive, i.e. if $\neg \neg a=a$ for every $a \in A$, then $\mathcal{A}$ is called an IMTL-algebra.

Thus, they are the variety of prelinear bounded commutative integral residuated lattices. Prelinearity property is enough to prove the following crucial facts:

Proposition $3.2([24])$.

- Every MTL-algebra is a subdirect product of linearly ordered MTL-algebras (which we will call MTL-chains).

\footnotetext{
${ }^{10}$ Examples of non-regular t-norms are those left-continuous t-norms with $a$-translations having infinitelymany discontinuity points introduced in [58].
} 
- Every variety of MTL-algebras is generated by its linearly ordered members (its chains).

In particular, the variety of all MTL-algebras is generated by the class of all MTL-chains, i.e. all linearly ordered bounded commutative integral residuated lattices.

Therefore, again, the problem of describing MTL-algebras reduces to that of understanding the structure of MTL-chains. However, as they contain in particular those based on left-continuous t-norms, this is a problem of amazing complexity and only some partial results are available.

The algebraic structures that will play now an analogous role to that of hoops in the BL case are the so-called semihoops.

Definition 3.3 ([26]). An algebra $\mathcal{A}=\langle A, \&, \rightarrow, \wedge, \overline{1}\rangle$ is a semihoop if:

- $\mathcal{A}=\langle A, \wedge, \overline{1}\rangle$ is an inf-semilattice with upper bound.

- $\langle A, \&, \overline{1}\rangle$ is a commutative monoid isotonic w.r.t. the inf-semilattice order $\leq$.

- For every $a, b \in A, a \leq b$ iff $a \rightarrow b=\overline{1}$.

- For every $a, b, c \in A, a \& b \rightarrow c=a \rightarrow(b \rightarrow c)$.

An operation $\vee$ is defined as: $a \vee b=((a \rightarrow b) \rightarrow b) \wedge((b \rightarrow a) \rightarrow a)$. A is called prelinear if for every $a, b, c \in A,(a \rightarrow b) \rightarrow c \leq((b \rightarrow a) \rightarrow c) \rightarrow c$. If $\mathcal{A}$ has a minimum element, then it is called a bounded prelinear semihoop (i.e. an MTL-algebra).

Notice that a prelinear semihoop $\mathcal{A}$ is a prelinear hoop if, and only if, it satisfies $a \&(a \rightarrow$ $b)=b \&(b \rightarrow a)$ for every $a, b \in A$. Every totally ordered semihoop is prelinear.

The notion of ordinal sum of totally ordered hoops is extended in the natural way to totally ordered semihoops, and the same with the notion of indecomposability. The following results from [72] extend the decomposability theorem to the whole class of MTL-chains:

Theorem 3.4. For every MTL-chain $\mathcal{A}$, there is a maximum decomposition as ordinal sum of indecomposable totally ordered semihoops, with the first one bounded.

Corollary 3.5. Let $\mathcal{A}$ be an MTL-chain. If the partition $\left\{[a]_{\sim} \mid a \in A \backslash\{\overline{1}\}\right\}$ given by the Archimedean classes gives a decomposition as ordinal sum, then it is the maximum one.

Definition 3.6. An MTL-chain is totally decomposable if, and only if, the partition given by its Archimedean classes gives a decomposition as ordinal sum.

Therefore, for all we have seen, finite BL-chains and BL-chains defined over the real unit interval (or, in general, over a complete chain) are totally decomposable, while BL-chains defined over the rationals are not.

Theorem 3.4 states a decomposition result for MTL-chains that in a sense generalizes the corresponding result for BL-chains. Unfortunately, here the result is by far not as useful as it was in the BL case. We can summarize the differences as follows:

1. The theorem for BL-chains gives a decomposition as ordinal sum of indecomposable Wajsberg hoops (thus, weakly cancellative hoops). In the MTL case, we can find indecomposable semihoops which are not weakly cancellative (for instance, the involutive MTL-chains). The variety generated by MTL-chains decomposable as ordinal sum of weakly cancellative semihoops has been studied in [72] and proved to be different from the variety of all MTL-chains. 
2. For BL-chains, the indecomposable Wajsberg hoops are known in the case of finite and real chains. There is also some partial knowledge on the indecomposable Wajsberg hoops over the rationals. Nevertheless, we do not have such kind of knowledge for indecomposable semihoops.

Another strategy to address the MTL problem has consisted in generalizing some construction/decomposition methods for left-continuous t-norms to the framework of MTL-chains. In [52] rotation and rotation-annihilation constructions are generalized to even weaker structures. For MTL-chains the corresponding definitions are the following:

Definition 3.7. Let $\mathcal{A}$ be a prelinear semihoop. The disconnected rotation of $\mathcal{A}$ is an algebra denoted $\mathcal{A}^{\circ}$ and defined as follows. Let $A \times\{0\}$ be a disjoint copy of $A$. For every $a \in A$ we write $a^{\prime}$ instead of $\langle a, 0\rangle$. Consider $\left\langle A^{\prime}=\left\{a^{\prime}: a \in A\right\}, \leq\right\rangle$ with the inverse order and let $A^{\circ}=A \cup A^{\prime}$. We extend these orderings to an order in $A^{\circ}$ by putting $a^{\prime}<b$ for every $a, b \in A$. Finally, we take the following operations in $\mathcal{A}^{\circ}: \overline{1}^{\mathcal{A}^{\circ}}=\overline{1}^{\mathcal{A}}, \overline{0}^{\mathcal{A}^{\circ}}=\left(\overline{1}^{\mathcal{A}}\right)^{\prime}, \wedge^{\mathcal{A}^{\circ}}$ is the minimum w.r.t. the ordering, $\vee \mathcal{A}^{\circ}$ is the maximum w.r.t. the ordering, and $\neg \mathcal{A}^{\circ}, \&^{\mathcal{A}^{\circ}}$ and $\rightarrow \mathcal{A}^{\circ}$ are respectively defined as

$$
\begin{aligned}
& \neg^{\mathcal{A}^{\circ}} a=\left\{\begin{array}{lll}
a^{\prime} & \text { if } & a \in A \\
b & \text { if } & a=b^{\prime} \in A^{\prime}
\end{array}\right. \\
& a \&^{\mathcal{A}^{\circ}} b= \begin{cases}a \&^{\mathcal{A}} b & \text { if } a, b \in A \\
\mathcal{A}^{\circ}\left(a \rightarrow \mathcal{A} \neg \mathcal{A}^{\circ} b\right) & \text { if } a \in A, b \in A^{\prime} \text { and } \neg^{\mathcal{A}^{\circ}} a \nsupseteq b \\
\mathcal{A}^{\circ}\left(b \rightarrow \mathcal{A} \neg^{\circ}{ }^{\circ} a\right) & \text { if } a \in A^{\prime}, b \in A \text { and } \neg^{\mathcal{A}^{\circ}} a \nsupseteq b \\
\overline{\mathcal{A}}^{\circ} & \text { otherwise }\end{cases} \\
& a \rightarrow \mathcal{A}^{\circ} b=\left\{\begin{array}{lll}
a \rightarrow{ }^{\mathcal{A}} b & \text { if } & a, b \in A \\
\mathcal{A}^{\circ}\left(a \mathcal{A}^{\mathcal{A}} \mathcal{A}^{\circ} b\right) & \text { if } & a \in A, b \in A^{\prime} \\
\overline{\mathcal{A}}^{\circ} & \text { if } & a \in A^{\prime}, b \in A \\
\mathcal{A}^{\circ} b \rightarrow \mathcal{A} \neg \mathcal{A}^{\circ} a & \text { if } & a, b \in A^{\prime} .
\end{array}\right.
\end{aligned}
$$

It is clear from this definition that the algebra introduced in Example 2.18 is actually isomorphic to the disconnected rotation of the cancellative hoop $\mathcal{H} \Pi$.

Definition 3.8. Let $\mathcal{A}$ be an MTL-algebra satisfying one of the following conditions:

- $\mathcal{A}$ does not have zero divisors. ${ }^{11}$

- $\exists c \in A$ such that $\forall a \in A$ zero divisor, $\neg a=c$.

Then, the connected rotation of $\mathcal{A}$ is denoted $\mathcal{A}^{\star}$ and defined as follows. Take $\left\langle A^{\prime}=\left\{a^{\prime}: a \in A, a \neq \overline{0}^{\mathcal{A}}\right\}, \leq\right\rangle$, a disjoint copy of $A \backslash\left\{\overline{0}^{\mathcal{A}}\right\}$ with the inverse order, and define $\neg \mathcal{A}^{\star} \overline{0}^{\mathcal{A}}=\overline{0}^{\mathcal{A}}$ and all the operations as in the disconnected rotation.

Proposition 3.9. Disconnected rotations are IMTL-algebras without negation fixpoint and connected rotations are IMTL-algebras with negation fixpoint.

\footnotetext{
${ }^{11} a \in A \backslash\{\overline{0}\}$ is a zero divisor if there exists $b \in A \backslash\{\overline{0}\}$ such that $a \& b=\overline{0}$.
} 
Observe that the rotation method for t-norms in the previous subsection is a particular case of this connected rotation, where the definition of operations was slightly more complicated due to the necessary rescalements of t-norms. These rotation methods have been used in $[78,79]$ where a theory of perfect and bipartite MTL-algebras is developed and perfect IMTLalgebras are characterized as disconnected rotations of prelinear semihoops, while perfect IMTL-algebras with an additional negation fixpoint are characterized as connected rotations of prelinear semihoops without zero divisors.

Definition 3.10. Let $\mathcal{A}$ be a prelinear semihoop and $\mathcal{B}$ be an IMTL-algebra such that $A \cap B=$ $\emptyset$. An algebra $\mathcal{C}$, called the disconnected rotation-annihilation of $\mathcal{A}$ and $\mathcal{B}$, is defined as follows. Let $\left\langle A^{\prime}=\left\{a^{\prime}: a \in A\right\}, \leq\right\rangle$ be a disjoint copy of $A$ as above (disjoint also with $B$ ) endowed with the inverse ordering and let $C=A \cup A^{\prime} \cup B$. The orderings are extended to $C$ by letting $a^{\prime}<b$ and $b<c$ for every $a, c \in A$ and every $b \in B$. Let $C^{+}=A, C^{0}=B$ and $C^{-}=A^{\prime}$. Finally, the following operations are defined on $\mathcal{C}: \overline{1}^{\mathcal{C}}=\overline{1}^{\mathcal{A}}, \overline{0}^{\mathcal{C}}=\left(\overline{1}^{\mathcal{A}}\right)^{\prime}, \wedge^{\mathcal{C}}$ is the minimum w.r.t. the order, $\vee^{\mathcal{C}}$ is the maximum w.r.t. the order, and $\neg^{\mathcal{C}}, \&^{\mathcal{C}}$ and $\rightarrow \mathcal{C}$ are respectively defined as

$$
\begin{aligned}
& \neg^{\mathcal{C}} a=\left\{\begin{array}{lll}
a^{\prime} & \text { if } & a \in A \\
b & \text { if } & a=b^{\prime} \in A^{\prime} \\
\neg^{\mathcal{B}} a & \text { if } & a \in B
\end{array}\right.
\end{aligned}
$$

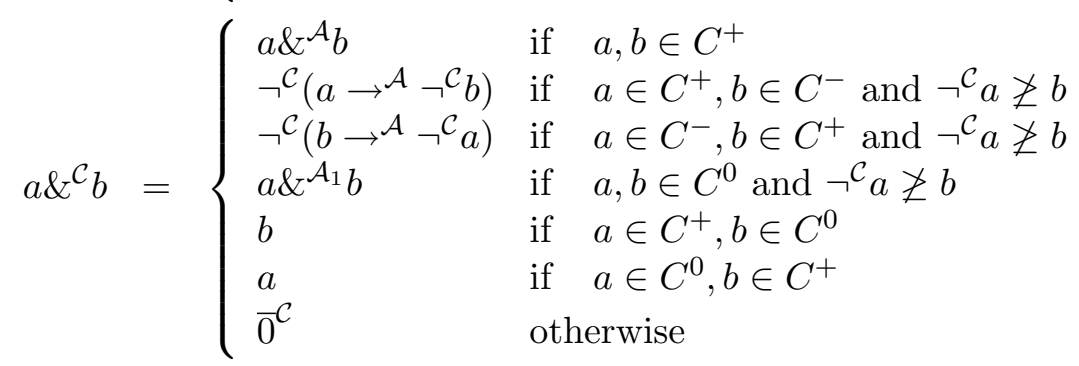

$$
\begin{aligned}
& a \rightarrow{ }^{\mathcal{C}} b=\left\{\begin{array}{lll}
a \rightarrow \rightarrow^{\mathcal{A}} b & \text { if } & a, b \in C^{+} \\
\left.{ }^{\mathcal{C}}\left(a \mathcal{A}^{\mathcal{A}}\right\urcorner^{\mathcal{C}} b\right) & \text { if } & a \in C^{+}, b \in C^{-} \\
\overline{1}^{\mathcal{C}} & \text { if } a \in C^{-}, b \in C^{+} \\
\neg^{\mathcal{C}} b \rightarrow^{\mathcal{A}} \neg^{\mathcal{C}} a & \text { if } a, b \in C^{-} \\
a \rightarrow \mathcal{A}_{1} & \text { if } & a, b \in C^{0} \text { and } a \not \leq b \\
\overline{1}^{\mathcal{C}} & \text { if } a, b \in C^{0} \text { and } a \leq b \\
b & \text { if } a \in C^{+}, b \in C^{0} \\
\overline{1}^{\mathcal{C}} & \text { if } a \in C^{0}, b \in C^{+} \\
\overline{1}^{\mathcal{C}} & \text { if } a \in C^{-}, b \in C^{0} \\
\neg^{\mathcal{C}} a & \text { if } a \in C^{0}, b \in C^{-} .
\end{array}\right.
\end{aligned}
$$

Definition 3.11. Let $a \mathcal{A}$ be a prelinear semihoop and $\mathcal{B}$ be an IMTL-algebra such that $A \cap B=\emptyset$. An algebra $\mathcal{C}$, called the connected rotation-annihilation of $\mathcal{A}$ and $\mathcal{B}$, is defined as follows. Let $\left\langle A^{\prime}=\left\{a^{\prime}: a \in A\right\}, \leq\right\rangle$ be a disjoint copy of $A$ as above (disjoint also with $B$ ) endowed with inverse ordering and let $C=A \cup A^{\prime} \cup B \backslash\left\{\overline{0}^{\mathcal{B}}, \overline{1}^{\mathcal{B}}\right\}$. We extend the orderings to $C$ by letting $a^{\prime}<b$ and $b<c$ for every $a, c \in A$ and every $b \in B$. Let $C^{+}=A$, $C^{0}=B \backslash\left\{\overline{0}^{\mathcal{B}}, \overline{1}^{\mathcal{B}}\right\}$ and $C^{-}=A^{\prime}$. Finally, the operations $\mathcal{C}$ are defined as in the disconnected rotation-annihilation. We will denote $\mathcal{C}$ as $\mathcal{A} \odot \mathcal{B}$. 
Proposition 3.12. Let $\mathcal{A}$ and $\mathcal{B}$ be a prelinear semihoop and an IMTL-algebra respectively. Then, the disconnected and connected rotation-annihilations of $\mathcal{A}$ and $\mathcal{B}$ are IMTL-algebras.

Observe that rotation-annihilation of t-norms as presented in the last subsection are a particular case of this connected rotation-annihilation. On the other hand, it is clear that in every connected rotation-annihilation $\mathcal{A} \odot \mathcal{B}$ the set $A$ is a proper filter of $\mathcal{A} \odot \mathcal{B}$ (recall that a filter in an MTL-algebra is a non-empty upwards directed set closed under the monoidal operation and the lattice infimum). Moreover, again by using Zorn's Lemma, we can prove that every IMTL-chain has a maximum decomposition as a connected rotation-annihilation (see [77] for more details):

Proposition 3.13. Let $\mathcal{A}$ be an IMTL-chain. Then, there is a maximum proper filter $F$ of $\mathcal{A}$ such that $\mathcal{A} \cong \mathcal{C} \odot \mathcal{B}$, where $\mathcal{C}$ is the semihoop determined by $F$ and $\mathcal{B}$ is the IMTL-chain determined by $(A \backslash(F \cup \neg F)) \cup\left\{\overline{0}^{\mathcal{A}}, \overline{1}^{\mathcal{A}}\right\}$, where $\neg F=\{\neg a \mid a \in F\}$.

Again the problem with such a decomposition is the lack of a description of the indecomposable elements.

On the other hand, as a positive result, MTL-chains enjoy the following powerful embedding property, which is not true for BL-chains.

Theorem 3.14. Every non-trivial countable MTL-chain is embeddable into an MTL-chain defined over the real unit interval.

The proof of this theorem is given by Jenei and Montagna by means of a method introduced in $[57,58]$ and refined in [74]. Actually it gives another method to construct left-continuous t-norms to be added to the list of the previous subsection:

5. Completion of a countable chain. Let $\mathcal{A}$ be a non-trivial countable MTL-chain. A standard MTL-chain $[0,1]_{*}$ and an embedding $h: \mathcal{A} \hookrightarrow[0,1]_{*}$ are built by following next steps:

- For every $a \in A, \operatorname{suc}(a)$ is either the successor of $a$ in the order of $\mathcal{A}$ if it exists or $\operatorname{suc}(a)=a$ otherwise.

- $B=\{\langle a, 1\rangle \mid a \in A\} \cup\left\{\langle a, q\rangle \mid \exists a^{\prime} \in A\right.$ such that $a \neq a^{\prime}$ and $\operatorname{suc}\left(a^{\prime}\right)=a$, $q \in \mathbb{Q} \cap(0,1)\}$.

- Consider the lexicographical order $\preceq$ on $B$.

- Define the following monoidal operation on $B$ :

$$
\langle a, q\rangle \circ\langle b, r\rangle= \begin{cases}\min _{\prec}\{\langle a, q\rangle,\langle b, r\rangle\} & \text { if } a \&^{\mathcal{A}} b=\min \{a, b\} \\ \left\langle a \&^{\mathcal{A}} b, 1\right\rangle & \text { otherwise. }\end{cases}
$$

- The ordered monoid $\left\langle A, \&^{\mathcal{A}}, \overline{1}^{\mathcal{A}}, \leq\right\rangle$ is embeddable into $\left\langle B, \circ,\left\langle\overline{1}^{\mathcal{A}}, 1\right\rangle, \preceq\right\rangle$ by mapping every $a \in A$ to $\langle a, 1\rangle$.

- $\mathcal{B}=\left\langle B, \circ,\left\langle\overline{1}^{\mathcal{A}}, 1\right\rangle, \preceq\right\rangle$ is a densely ordered countable monoid with maximum and minimum, so it is isomorphic to a monoid $\mathcal{B}^{\prime}=\left\langle[0,1]^{\mathbb{Q}}, o^{\prime}, 1, \preceq^{\prime}\right\rangle$. Obviously, $\left\langle A, \&^{\mathcal{A}}, \overline{1}^{\mathcal{A}}, \leq\right\rangle$ is also embeddable into $\mathcal{B}^{\prime}$. Let $h$ be such embedding. Moreover, restricted to $h[A]$, the residuum of $\circ^{\prime}$ exists, call it $\Rightarrow$, and $h(a) \Rightarrow h(b)=h\left(a \rightarrow^{\mathcal{A}} b\right)$. 
- $\mathcal{B}^{\prime}$ is completed to $[0,1]$ by defining:

$$
\forall \alpha, \beta \in[0,1] \quad \alpha * \beta=\sup \left\{x \circ^{\prime} y \mid x \leq \alpha, y \leq \beta, x, y \in[0,1]^{\mathbb{Q}}\right\} .
$$

- $*$ is a left-continuous t-norm, so it defines a standard MTL-algebra $[0,1]_{*}$, and $h$ is the desired embedding.

This method allows us to build an extension of the monoidal operation in any countable MTL-chain to a left-continuous t-norm over the real unit interval. Thus all continuous and left-continuous t-norms over a countable chain (for example the rationals) are restrictions of a left-continuous t-norm over the real unit interval. Notice that, in particular, the way of extending a left-continuous t-norm on the rationals to the reals as it is done in the last step of the above completion method is the only possible way that preserves the left-continuity. In fact, the above method says that any left-continuous t-norm over the rational unit interval (that can have residuum or not) ${ }^{12}$ is a restriction of a left-continuous t-norm over the real unit interval (that always has residuum), and thus it is a restriction of a MTL-chain over the real unit interval.

As a consequence we obtain the following corollary.

Corollary 3.15. Every MTL-chain defined over the rational unit interval is the restriction of an MTL-chain defined over the real unit interval.

However, the above completion method may not preserve many other properties the initial MTL-chain may satisfy. For example, divisibility or cancellativity is not preserved by the completion method. As an example consider the chain in Example 2.18. The completion of this chain in the real interval introduces a new element separating the two non-trivial Archimedean components, say $\alpha$. Then for every $x>\alpha, x * \alpha=\alpha$ and $\alpha * \alpha=0$, and thus cancellativity and divisibility do not hold any longer. Thus, as we have already mentioned before, not all BL-chains over the rationals are subalgebras of a BL-chain over the reals, and the same holds for cancellative MTL-chains satisfying (see in [23] an example of a cancellative MTL-chain (not BL) that its completion by the Jenei-Montagna method does not give a cancellative MTL-chain).

As regards to finite MTL-chains, Theorem 3.14 guarantees that, in particular, all finite chains are embeddable into an MTL-chain over the real unit interval. Moreover, one can show that this method provides an embedding of any finite MTL-chain into a MTL-chain over the rationals as well. Remember that for finite BL-chains, based on the knowledge about their structure, we have proved at the end of Section 2.2 that all of them are subalgebras of a BL-chain over the rationals and also a subalgebra of a BL-chain over the reals (a restriction of a continuous t-norm).

Summing up, the embedding results show that left-continuous t-norms over the real unit interval are so complex and rich that, in particular, contain as subalgebras all MTL-chains defined over finite, rational or, in general, countable chains. So solving the problem of the description of left-continuous t-norms would be enough to understand countable MTL-chains as well.

\footnotetext{
${ }^{12}$ See Example 4.3 for an example of a left-continuous t-norm over the rational unit interval that has no residuum.
} 


\section{About continuity and left-continuity conditions over the or- der topology}

An obvious way to generalize continuity and left-continuity on a chain could be the use of the order topology defined over the chain. This is something studied in different papers but it is in [7] where the authors studied the relation between the continuity with respect to the order topology and the existence of residuum on a chain. In particular, it is shown there the following:

- If a t-norm over a chain has residuum, then it is left-continuous with respect to order topology. The converse is not true in general (see Example 4.1), but at least it holds for complete chains.

- If a t-norm over a chain has residuum and satisfies the divisibility condition, then it is continuous with respect to the order topology. The converse result is true only when the chain is complete and dense (see Example 4.2).

Next we give some illustrative examples:

Example 4.1. Take $Y=\left\{\frac{1}{2}+\frac{1}{2^{n}} \mid n \in \omega, n>0\right\}$ and $C=\{0\} \cup\left[\frac{1}{5}, \frac{1}{4}\right) \cup Y$ and define the following operation on the chain $C$ :

$$
x * y= \begin{cases}\frac{(2 x-1)(2 y-1)+1}{2}, & \text { if } x, y \in Y \\ \frac{1}{5}, & \text { if } x \in\left[\frac{1}{5}, \frac{1}{4}\right) \text { and } y \in Y \backslash\{1\}, \text { or } y \in\left[\frac{1}{5}, \frac{1}{4}\right) \text { and } x \in Y \backslash\{1\} \\ 0, & \text { if } x, y \in\left[\frac{1}{5}, \frac{1}{4}\right) \text { or } \min \{x, y\}=0 \\ x, & \text { if } y=1 \\ y, & \text { if } x=1\end{cases}
$$

An easy computation shows that $*$ is commutative, associative, ${ }^{13}$ monotonic, 1 is neutral and it is left-continuous because it is so on the interval $\left[\frac{1}{5}, \frac{1}{4}\right)$. But it has no residuum since if $x \in Y \backslash\{1\}$ and $y \in\left[\frac{1}{5}, \frac{1}{4}\right)$, then

$$
\max \{c \in C \mid c * x \leq y\}=\max \left\{\left[\frac{1}{5}, \frac{1}{4}\right)\right\}
$$

does not exist in $C$.

Finally observe that the same example could be defined taking the same set $C$ but with only the rationals on the interval $\left[\frac{1}{5}, \frac{1}{4}\right)$, and in that case the chain $C$ would be countable.

Example 4.2. Let $C$ be the chain of five elements, $0<a<b<c<1$ and let $*$ be the binary operation on $C$ defined as follows:

\begin{tabular}{|c||c|c|c|c|c|}
\hline$*$ & 0 & $a$ & $b$ & $c$ & 1 \\
\hline \hline 0 & 0 & 0 & 0 & 0 & 0 \\
\hline$a$ & 0 & $a$ & $a$ & $a$ & $a$ \\
\hline$b$ & 0 & $a$ & $a$ & $a$ & $b$ \\
\hline$c$ & 0 & $a$ & $a$ & $c$ & $c$ \\
\hline 1 & 0 & $a$ & $b$ & $c$ & 1 \\
\hline
\end{tabular}

\footnotetext{
${ }^{13}$ Take into account that $*$ restricted to $Y$ is the product generated by one element and sent back to the set $Y$.
} 
Since $C$ is finite, it is of course complete as well and the operation $*$ is continuous with respect to the order topology, and thus it has residuum. Nevertheless it does not satisfy the divisibility property since it holds that

$$
c *\left(c \rightarrow_{*} b\right)=c * b=a \neq \min \{b, c\} .
$$

Recalling that a countable densely ordered chain is order-isomorphic to the rationals, the next example shows a left-continuous t-norm over the rational unit interval that has no residuum. This is in accordance with Jenei-Montagna's method where the extension of the monoidal operation in a countable MTL-chain to a left-continuous t-norm over the rationals may not have residuum, although its extension over the reals (preserving left-continuity) always does.

Example 4.3. Let $C=\left\{\frac{k}{2^{n}} \mid n \in \omega \backslash\{0\}, 0 \leq k \leq 2^{n}\right\}$ and take the product $t$-norm there. It is clear that $C$ is a countable and densely ordered set, and the product operation is closed in this set. This operation is clearly continuous on $C$ but it does not has residuum, since, for example, $\max \left\{\frac{k}{2^{n}} \in C \mid \frac{k}{2^{n}} \cdot \frac{3}{4} \leq \frac{1}{2}\right\}$ does not exist. Since $C$ is order-isomorphic to the rationals, the induced operation on $[0,1] \cap \mathbb{Q}$ is continuous t-norm over the rationals and has no residuum.

The above examples show that the notion of continuity and left-continuity with respect to the order topology is not well related with the residuation property. On the other hand, another way to generalize the concept of continuity of a t-norm $*$ on a chain $C$ is trying to keep the property that the image of $C$ by the map $f_{x}(y)=x * y$ is the closed interval $[0, x]$ in $C$. This property is actually equivalent to being divisible in the sense that if $a<b$ there exists $c$ such that $a=b * c$ (cf. Proposition 2.1 for t-norms). But, as in the case of t-norms, it is well known (see e.g. [37]) that this property over chains is equivalent to the so-called divisibility equation $x *\left(x \rightarrow_{*} y\right)=\min \{x, y\}$ that characterizes BL-algebras. Thus we think that taking BL-chains as generalizations of continuous t-norms is fully justified.

\section{Conclusions}

In this paper we have defended the notions of BL-chains and MTL-chains as adequate generalizations of continuous and left-continuous t-norms respectively, arisen from the study of algebraic semantics for fuzzy logics over chains with a monoidal residuated operation.

When requiring divisibility property to ensure continuity of the operation over $[0,1]$, we have dealt with BL-chains and continuous t-norms. The latter are completely described by means of the classical decomposition in ordinal sum of three basic components. We have seen analogous structural results that apply to all BL-chains which in particular allow to describe all finite BL-chains. However, they are not enough to determine the structure of BL-chains over the rational unit interval mainly because there is a very large set of them that are not isomorphic to restrictions of BL-chains over the unit real interval. It remains as an open problem the description of the indecomposable Wajsberg hoops over the rationals in order to obtain a full characterization of BL-chains over the rational unit interval.

In contrast, when divisibility property is dropped and one deals with left-continuous tnorms and MTL-chains, the situation changes dramatically. On the one hand, thanks to a completion construction that shows that all non-trivial countable MTL-chains are embeddable into one defined over the reals by a left-continuous t-norm (which is not true for BL-chains 
and continuous t-norms), the problem seems to be simpler as a description of left-continuous t-norms would in particular yield a description of MTL-chains over rationals and over finite sets. However, on the other hand, one realizes that the structure of left-continuous t-norms is so wild and rich that such a goal is still out of hand. As we have reviewed, several valuable approaches to that problem have been made mainly by giving construction-decomposition methods for distinguished families of left-continuous t-norms. We have also discussed their influence to the general study of MTL-chains.

Another interesting topic about t-norms over chains is their equational characterization. We would like to point out for interested readers that there are several papers devoted to this topic. In the continuous case the main reference is [25]. In that paper it is proved that every standard BL-chain (a BL-chain $[0,1]_{*}$ defined by a continuous t-norm $*$ and its residuum) generates a finitely axiomatizable variety, and an algorithm is provided to find such an axiomatization. But, is it the same to characterize the variety generated by $[0,1]_{*}$ than characterizing the t-norm * itself? In other words, how many standard BL-chains generate the same variety? The answer to this question is also indirectly solved in the cited paper since it is shown there that each t-norm whose decomposition as ordinal sum of $\mathrm{E}, \mathrm{G}$ and $\Pi$ components is finite defines a different variety. But the result is not true if the decomposition has infinitely-many components.

As a consequence when the t-norm has a finite decomposition as ordinal sum of the three main continuous t-norms, the equations characterizing the variety generated by the corresponding standard chain, characterize the t-norm as well, ${ }^{14}$ but this is not true for the infinite case.

The problem for left-continuous t-norms is far from being solved and only a few cases have been axiomatized. The main partially solved case is that of Weak Nilpotent Minimum (WNM) t-norms. The t-norms in this family are the result of the annihilation of the minimum t-norm by a negation function (when the negation function is involutive we obtain, up to isomorphism, Fodor's Nilpotent Minimum t-norm). In [80] the equations characterizing the variety generated by the standard chain defined by a WNM t-norm are given only when the WNM t-norm satisfies the so-called Finite Partition Property, and in this case they also characterize the t-norm. Moreover, the variety generated by a finite WNM-chain is also equationally characterized. The particular case of NM and its subvarieties have been fully described in [36]. Other cases have been partially studied but so far there is not a systematic study of the equational characterization of left-continuous t-norms.

As interesting open problems for further research we point out that, similarly to what has been done for the rotation and rotation-annihilation) constructions, it would be desirable to (i) generalize the triple rotation method to obtain IMTL-chains and algebraically characterize their structure (as disconnected rotations were characterized as perfect algebras in [78]), and (ii) generalize the class of regular left-continuous t-norms from [87] to some notion of regular MTL-chains that would still be a generating family for the whole variety with, hopefully, a describable algebraic structure.

\section{Acknowledgments}

We want to thank Vicenzo Marra for his helpful comments on group theory and the anonymous referees for their many comments and remarks that helped us improving much the first

\footnotetext{
${ }^{14}$ In the sense that there is only one t-norm up to isomorphism satisfying these equations.
} 
version of this paper. Moreover, we acknowledge partial support of the research projects MULOG2 (TIN2007-68005-C04) and ESF Eurocores-LogICCC / MICINN project (FFI200803126-E/FILO). The first author also acknowledges partial support from the grant 2006-BPA-10043 of the Departament d'Educació i Universitats of the Generalitat de Catalunya.

\section{References}

[1] P. Aglianò, I. M. A. Ferreirim and F. Montagna. Basic hoops: an algebraic study of continuous t-norms, Studia Logica 87 (2007) 73-98.

[2] P. Aglianò and F. Montagna. Varieties of BL-algebras I: general properties, Journal of Pure and Applied Algebra 181 (2003) 105-129.

[3] C. Alsina, E. Trillas and L. Valverde. On some logical connectives for Fuzzy Set Theory, J. Math. An. and Appl. 93 (1983) 15-26.

[4] C. Alsina, M. Frank and B. Schweizer. Associative Functions: Triangular Norms and Copulas, World Scientific, 2006.

[5] W.J. Blok and I.M.A. Ferreirim. Hoops and their implicational reducts, Algebraic Logic, Banach Center Pub. 28, Warsaw 1993.

[6] W.J. Blok And D. Pigozzi. Algebraizable logics, Memoirs of the American Mathematical Society 396, vol 77, 1989.

[7] D. Boixader, F. Esteva and L. Godo. On the continuity of t-norms on bounded chains. In Proceedings of the 8th IFSA World Congress IFSA'99, August 1999, Taipei (Taiwan), pp. 476-479.

[8] S. Burris and H.P. Sankappanavar. A course in Universal Algebra, Springer Verlag, New York, 1981.

[9] C.C. Chang. Algebraic analysis of many valued logics, Transactions of the American Mathematical Society 88 (1958) 456-490.

[10] C.C. Chang. A new proof of the completeness of the Lukasiewicz axioms, Transactions of the American Mathematical Society 93 (1959) 74-80.

[11] A. Ciabattoni, F. Esteva and L. Godo. T-norm based logics with $n$-contraction, Neural Network World 5 (2002) 441-452.

[12] R. Cignoli, F. Esteva, L. Godo and F. Montagna. On a class of left-continuous t-norms, Fuzzy Sets and Systems 131 (2002) 283-296.

[13] R. Cignoli, F. Esteva, L. Godo And A. Torrens. Basic Fuzzy Logic is the logic of continuous t-norms and their residua, Soft Computing 4 (2000) 106-112.

[14] R. Cignoli And A. Torrens, An Algebraic Analysis of Product Logic, Multiple Valued Logic 5 (2000) 45-65. 
[15] P. Cintula, F. Esteva, J. Gispert, L. Godo, F. Montagna and C. Noguera. Distinguished algebraic semantics for t-norm based fuzzy logics: methods and algebraic equivalencies, Annals of Pure and Applied Logic 160 (2009) 53-81.

[16] A.H. Clifford. Naturally totally ordered commutative semigroups, Amer. L. Math. 76 (1954) 631-646.

[17] A.C. Climescu. Sur l'équation fonctionelle de l'associativité, Bull. École Polytechn. Iassy 1 (1946) 1-16.

[18] B. De Baets And R. Mesiar. Triangular norms on product lattices, Fuzzy Sets and Systems 104 (1999) 61-75.

[19] D. Dubois and H. Prade. New Results about Properties and Seman tics of Fuzzy Set-theoretic Operators, First Symposium on Policy Analysis and Information Systems Durham, North Caroline, USA, (1979) 167-174.

[20] D. Dubois And H. Prade. Fuzzy Sets and Systems - Theory and Applications, New York: Academic Press, 1980.

[21] M. Dummett. A propositional calculus with denumerable matrix, The Journal of Symbolic Logic 24 (1959) 97-106.

[22] F. Esteva And X. Domingo. Sobre negaciones fuertes y débiles en [0,1], Stochastica 4 (1980) 141-166. (In Spanish)

[23] F. Esteva, J. Gispert, L. Godo and F. Montagna. On the standard and rational completeness of some axiomatic extensions of the monoidal t-norm logic, Studia Logica 71(2) (2002) 199-226.

[24] F. Esteva And L. Godo. Monoidal t-norm based logic: Towards a logic for leftcontinuous t-norms, Fuzzy Sets and Systems 124 (2001) 271-288.

[25] F. Esteva, L. Godo and F. Montagna. Equational Characterization of the Subvarieties of BL Generated by t-norm-algebras, Studia logica 76 (2004) 161-200.

[26] F. Esteva, L. Godo, P. Hájek and F. Montagna. Hoops and Fuzzy Logic, Journal of Logic and Computation 13 (2003) 531-555.

[27] F. Esteva, L. Godo and C. Noguera. Real, rational and finite-chain semantics for fuzzy logics. In Proceedings of the XIII Congreso Español sobre Tecnologías y Lógica Fuzzy (ESTYLF 2006), 20-22 September, Ciudad Real (Spain), pp. 83-88, 2006.

[28] F. Esteva, L. Godo and C. Noguera. First-order t-norm based fuzzy logics with truth-constants: distinguished semantics and completeness properties, Annals of Pure and Applied Logic 161 (2009) 185-202.

[29] I. M. A. Ferreirim. On varieties and quasivarieties of hoops and their reducts, Ph.D. dissertation, University of Illinois at Chicago, 1992.

[30] T. Flaminio. Strong non-standard completeness for fuzzy logics, Soft Computing 12 (2007) 321-333. 
[31] J. Fodor. Nilpotent minimum and related connectives for fuzzy logic, Proceedings of FUZZ-IEEE'95, 1995, pp. 2077-2082.

[32] L. Fuchs. Abelian groups, Pergamon Press, New York, 1960.

[33] L. Fuchs. Partially Ordered Algebraic Systems, Pergamon Press, Oxford, London, New York, Paris, 1963.

[34] H. Gaitán. The number of simple one-generated bounded commutative BCK-chains Math. Japonica, Vol. 38, No. 3, pp. 483-486, 1993.

[35] N. Galatos, P. Jipsen, T. Kowalski and H. Ono. Residuated Lattices: An Algebraic Glimpse at Substructural Logics, volume 151 of Studies in Logic and the Foundations of Mathematics. Elsevier, Amsterdam, 2007.

[36] J. Gispert. Axiomatic extensions of the nilpotent minimum logic, Reports on Mathematical Logic 37 (2003) 113-123.

[37] A.M.W. GLass. Partially ordered groups, Series in Algebra vol. 7, World Scientific, 1999.

[38] K. GöDEL. Zum intuitionistischen Aussagenkalkül, Anzeiger Akademie der Wissenschaften Wien, Math. naturwiss. Klasse 69 (1932) 65-66.

[39] L. Godo And C. Sierra. A New Approach to Connective Generation in the Framework of Expert Systems using Fuzzy Logic. In Proceedings of the 18th IEEE International Symposium on Multiple-Valued Logic (ISMVL'88), Palma, Spain, pp. 157-163, 1988.

[40] K. Goodearl. Partially ordered Abelian groups with interpolation, Mathematical Surveys and Monographs vol. 20, AMS, Providence, 1986.

[41] P. HÁseK. Metamathematics of fuzzy logic, Trends in Logic vol. 4, Kluwer, Dordrecht, 1998.

[42] P. HÁJeK. Basic fuzzy logic and BL-algebras, Soft Computing 2 (1998) 124-128.

[43] P. HÁJek, L. Godo and F. Esteva. A complete many-valued logic with productconjunction, Archive for Mathematical Logic 35 (1996) 191-208.

[44] U. HöHLE. Minkowski functionals of L-fuzzy sets, First Symposium on Policy Analysis and Information Systems Durham, North Caroline, USA, pp. 178-186, 1979.

[45] R. HorČík, C. Noguera And M. Petrík. On n-contractive fuzzy logics, Mathematical Logic Quarterly 53 (2007) 268-288.

[46] S. JENEI. New family of triangular norms via contrapositive symmetrization of residuated implications, Fuzzy Sets and Systems 110 (1999) 157-174.

[47] S. JenEI. Structure of left-continuous triangular norms with strong induced negations, (I) Rotation construction, Journal of Applied Non-Classical Logics 10 (2000) 83-92.

[48] S. JenEI. Structure of left-continuous triangular norms with strong induced negations, (II) Rotation-annihilation construction, Journal of Applied Non-Classical Logics 10 (2001) 351-366. 
[49] S. JENEI. A note on the ordinal sum theorem and its consequence for the construction of triangular norms, Fuzzy Sets and Systems 126 (2002) 199-205.

[50] S. JenEI. Structure of left-continuous triangular norms with strong induced negations, (III) Construction and decomposition, Fuzzy Sets and Systems 128 (2002) 197-208.

[51] S. JenEI. Structure of Girard monoids on [0,1], in: S. E. Rodabaugh, E. P. Klement (Eds.), Topological and Algebraic Structures in Fuzzy Sets. A Handbook of Recent Developments in the Mathematics of Fuzzy Sets, Kluwer Academic Publishers, Dordrecht, 2003, 277-308.

[52] S. JENEI. On the structure of rotation-invariant semigroups, Archive for Mathematical Logic 42 (2003) 489-514.

[53] S. JenEI. On the determination of left-continuous t-norms and continuous archimedean t-norms on some segments, Aequationes Mathematicae 70 (2005) 177-188.

[54] S. JeneI. On the convex combination of left-continuous t-norms, Aequationes Mathematicae 72 (2006) 47-59.

[55] S. JenEI. On reflection invariance of residuated chains, Annals of Pure and Applied Logic 161 (2009) 220-227.

[56] S. Jenei And B. De Baets. On the direct decomposability of t-norms on product lattices, Fuzzy Sets and Systems 139 (2003) 699-707.

[57] S. Jenei and F. Montagna. A proof of standard completeness for Esteva and Godo's logic MTL, Studia Logica 70 (2002) 183-192.

[58] S. Jenei and F. Montagna. A general method for constructing left-continuous tnorms, Fuzzy Sets and Systems 136 (2003) 263-282.

[59] S. Jenei and F. Montagna. On the continuity points of left-continuous t-norms, Archive for Mathematical Logic 42 (2003) 797-810.

[60] E.P. Klement. Some remarks on t-norms, fuzzy-algebras and fuzzy-measures, Proceedings of 2nd International Linz Seminar on Fuzzy Set Theory E.P. Klement (ed.), (1980) $125-142$.

[61] E.P. Klement, R. Mesiar and E. PaP. Triangular Norms, Kluwer Academic Publishers, Dordrecht, 2000.

[62] V.M. Kopytov and N. Ya. Medvedev. The theory of Lattice-Ordered Groups. Kluwer, 1994.

[63] C.H. Ling. Representation of associative functions, Publ. Math. Debrecen 12 (1965) 189-212.

[64] J. Łukasiewicz And A. TARski. Untersuchungen ber den Aussagenkalkl. Comptes Rendus de la Societé des Sciences et des Lettres de Varsovie, cl. iii 23 (1930) 1-21.

[65] K.C. Maes And B. De Baets. On the structure of left-continuous t-norms that have a continuous contour line, Fuzzy Sets and Systems 158 (2007) 843-860. 
[66] K.C. Maes and B. De Baets. The triple rotation method for constructing t-norms, Fuzzy Sets and Systems 158 (2007) 1652-1674.

[67] K.C. Maes and B. De Baets. Rotation-invariant t-norms: where triple rotation and rotation-annihilation meet, Fuzzy Sets and Systems 160 (2009) 1998-2016.

[68] K.C. Maes and A. Mesiarová-Zemánková. Cancellativity properties for t-norms and t-subnorms, Information Sciences 179 (2009) 1221-1233.

[69] G. Mayor and J. Torrens. On a class of operators for expert systems, International Journal of Intelligent Systems 8 (1993) 771-778.

[70] G. Mayor and J. TorRens. Triangular norms on discrete settings. In Logical, Algebraic, Analytic, and Probabilistic Aspects of Triangular Norms, E.P. Klement, R. Mesiar (Eds.), Elsevier, Amsterdam, 2005, pp. 189-230.

[71] K. Menger. Statistical metrics, Proc. Nat. Acad. Sci. USA 8 (1942) 535-537.

[72] F. Montagna, C. Noguera and R. HorČík. On weakly cancellative fuzzy logics, Journal of Logic and Computation 16 (2006) 423-450.

[73] F. Montagna and C. Noguera. Arithmetical complexity of first-order predicate fuzzy logics over distinguished semantics, Journal of Logic and Computation, in press.

[74] F. Montagna and H. Ono. Kripke semantics, undecidability and standard completeness for Esteva and Godo's logic MTL $\forall$, Studia Logica 710 (2002) 227-245.

[75] P.S. Mostert And A.L. Shields. On the structure of semigroups on a compact manifold with boundary, Annals of Math. 65 (1957) 117-143.

[76] D. Mundici. Interpretation of AF $C^{*}$-algebras in Łukasiewicz Sentential Calculus, Journal of Functional Analysis 65 (1986) 15-63.

[77] C. Noguera. Algebraic Study of Axiomatic Extensions of T-Norm Based Fuzzy Logics. Monografies de l'Institut d'Investigació en Intel-ligència Artificial, vol. 27, Bellaterra, 2007.

[78] C. Noguera, F. Esteva and J. Gispert. Perfect and bipartite IMTL-algebras and disconnected rotations of prelinear semihoops, Archive for Mathematical Logic 44 (2005) 869-886.

[79] C. Noguera, F. Esteva and J. Gispert. On some varieties of MTL-algebras, Logic Journal of the IGPL 13 (2005) 443-466.

[80] C. Noguera, F. Esteva and J. Gispert. On triangular norm based axiomatic extensions of the Weak Nilpotent Minimum logic, Mathematical Logic Quarterly 54 (2008) $387-409$.

[81] P. Perny and B. Roy. The use of fuzzy Outranking Relations in Preference Modelling, Fuzzy sets and Systems 49 (1992) 33-53.

[82] M. Petrík And P. SARKoci. Associativity of triangular norms in light of web geometry. Proceedings of IFSA-EUSFLAT 2009. 
[83] B. Schweizer And A. Sklar. Statistical metric spaces, Pacific J. Math. 10 (1960) 313-334.

[84] B. Schweizer And A. Sklar. Associative functions and abstract semigroups, Publ. Math. Debrecen 10 (1963) 69-81.

[85] E. TRILlas. Sobre funciones de negación en la teoría de conjuntos difusos, Stochastica 3 (1979) 47-60.

[86] E. Trillas and L. Valverde. On some functionally expressable implications for fuzzy set theory, Proceedings of the 3rd International Seminar on Fuzzy Set Theory 1981, Linz, 173-190.

[87] T. Vetterlein. Regular left-continuous t-norms, Semigroup Forum 77 (2008) 339-379.

[88] Z.H. YI, F. QIN AND W.C. LI. Generalizations to the constructions of t-norms: rotation(-annihilation) construction, Fuzzy Sets and Systems 159 (2008) 1619-1630.

[89] L. A. Zadeh. Fuzzy sets, Information Control 8 (1965) 338-353.

[90] D. Zhang. Triangular norms on partially ordered sets, Fuzzy Sets and Systems 153 (2) (2005) 195-209. 